\title{
Granular suspension avalanches. II. Plastic regime
}

\author{
Nicolas Andreini, Christophe Ancey, and Gaël Epely-Chauvin ${ }^{\text {a) }}$ \\ École Polytechnique Fédérale de Lausanne, Écublens, 1015 Lausanne, Switzerland
}

(Received 7 August 2012; accepted 25 January 2013; published online 6 March 2013)

\begin{abstract}
We present flume experiments showing plastic behavior for perfectly density-matched suspensions of non-Brownian particles within a Newtonian fluid. In contrast with most earlier experimental investigations (carried out using coaxial cylinder rheometers), we obtained our rheological information by studying thin films of suspension flowing down an inclined flume. Using particles with the same refractive index as the interstitial fluid made it possible to measure the velocity field far from the wall using a laser-optical system. At long times, a stick-slip regime occurred as soon as the fluid pressure dropped sufficiently for the particle pressure to become compressive. Our explanation was that the drop in fluid pressure combined with the surface tension caused the flow to come to rest by significantly increasing flow resistance. However, the reason why the fluid pressure diffused through the pores during the stick phases escaped our understanding of suspension rheology. (c) 2013 American Institute of Physics. [http://dx.doi.org/10.1063/1.4793720]
\end{abstract}

\section{INTRODUCTION}

Fluid plasticity is closely related to the concept of yield stress, i.e., the minimum stress necessary to bring about an unbounded deformation of the material. In colloidal systems, this concept is now fairly well understood. ${ }^{1}$ At low solids fractions, yielding results from the breakdown of the weak links between flocs, while at high solids fractions, yielding results from the rupture of inter-particle bonds and the resistance to the deformation of networks. There is still much debate about yield stress in suspensions composed of non-Brownian coarse particles. Several authors have documented the existence of a yield stress in the limit of vanishing shear rate. ${ }^{2-4}$ During the early attempts to model yield stress in non-colloidal systems, a popular idea was that the yield stress arises from the dependence of the maximum solid concentration on the shear stress. ${ }^{2,5}$ Another explanation lies in the process of particle settling where frictional contacts between particles generate Coulomb friction on the bulk scale. ${ }^{6,7}$ Using magnetic resonance imaging, Ovarlez, Bertrand, and Rodts ${ }^{8}$ measured the velocity and concentration profiles inside a wide-gap coaxial-cylinder rheometer. They observed an apparent yield in the macroscopic rheometric measurements even though the particle-scale rheological behavior was viscous. Elaborating the experimental procedure, Fall et al. ${ }^{9}$ found that a slight density contrast was necessary for an apparent yield stress to arise. For perfectly density-matched suspensions, they observed no plastic behavior except for very high concentrations, on which the combined action of surface tension and dilatancy imposed steric constraints.

This paper follows on from another paper (hereafter referred to as Paper I), ${ }^{61}$ which investigated the flow behavior of fixed volumes of particle suspension flowing down a flume. The focus was on very concentrated particle suspensions comprising neutrally buoyant particles, i.e., whose solids fraction exceeded 0.57. Macroscopic measurements (e.g., the front position with time) showed that at short times, the suspension flowed like a viscous fluid. At a critical time, there was a sudden change of behavior as the flow reached a new regime characterized by the development of fractures and intermittent motion.

The objective of this paper is to investigate this long-term behavior of concentrated particle suspensions and infer some rheological information. Here we take advantage of the recent advances

a) Deceased. 
in optical and visualization methods,${ }^{10}$ which make it possible to supplement macroscopic measurements by taking local measurements inside the flowing material. In contrast with results obtained using conventional rheometers, we show that even in an apparently steady-state flow, plastic behavior appears after a finite time and is strongly correlated with (perhaps even controlled by) pore-fluid pressure, i.e., the interstitial fluid pressure in the pores of the granular matrix. After a very long time, there is an abrupt transition from steady creeping flow to intermittent motion, characterized by repetitive stick-slip cycles. We begin with a short description of the experimental protocol (see Sec. II). The details can be found in Paper I. ${ }^{61}$ We then describe the features of the fracture and plastic regimes (see Sec. III). We finally comment on the interpretation in terms of rheological behavior (see Sec. IV). A key element in the understanding of the observed flow pattern was the diffusion of interstitial fluid pressure. In the Appendix, we outline recent theories that account for the coupling between the solid disperse phase and the continuous interstitial fluid. As will become apparent later, none of these theories is sufficient to explain all of our observations.

\section{EXPERIMENTAL}

We studied the rheological behavior of suspensions composed of neutrally buoyant particles immersed in a Newtonian solution. We explored the 0.30-0.61 range of solids fraction. We used poly(methyl methacrylate) (PMMA) particles of uniform size distribution from $15 \mu \mathrm{m}$ to $175 \mu \mathrm{m}$ (mean diameter $110 \mu \mathrm{m}$ ). Using a sieving machine with a $180 \mu \mathrm{m}$ sealed sieving stack, we narrowed the size distribution (mean diameter $190 \mu \mathrm{m}$, standard deviation $60 \mu \mathrm{m}$ ). The Newtonian solution was a mixture of fluids called trimix of viscosity $0.124 \mathrm{~Pa}$ s at $20^{\circ} \mathrm{C}$, composed of $50 \%$ Triton X100, $28 \%$ 1,6-Dibromohexane, and 22\% UCON oil (75-H450 oil from Dow Chemicals). The particles and fluids had the same refractive index to within $10^{-4}$. The density mismatch between the fluid and solid phases was zero to within $5 \times 10^{-4} \mathrm{~g} \mathrm{~cm}^{-3}$. The final bulk density was $\rho=1.184 \pm 0.0005 \mathrm{~g} / \mathrm{cm}^{3}$, the refractive index was $1.48850 \pm 0.00025$ for a wavelength of $532 \mathrm{~nm}$ and at $20^{\circ} \mathrm{C}$. The surface tension was $\gamma=33 \pm 5 \mathrm{mN} \mathrm{m}^{-1}$. The random close packing $\phi_{R C P}$ was $\phi_{R C P}=0.655 \pm 0.02$ independently of the particle distribution. The permeability of the unsieved material was $k=4 \times 10^{-12} \mathrm{~m}^{2}$ (a much lower value than that given by the Kozeny-Carman equation for monosized particles).

Experiments were conducted in a $3.5-\mathrm{m}$ long, $10-\mathrm{cm}$ wide flume, equipped with a 10-liter reservoir in its upper part. Flow depths and velocities were measured using high-speed cameras (operated at $200 \mathrm{~Hz}$ ) and particle image velocimetry techniques. Fluid pressures were measured at the base of the flume using differential piezoelectric sensors (Honeywell DC001NDC4). A dataacquisition device (model USB 6221 from National Instruments) recorded the pore-pressure signals at $500 \mathrm{~Hz}$, the output signal of the cameras (to determine whether the cameras were acquiring frames), and position of the sluice gate (down or up). Each piezoelectric sensor was connected by two 4-mm tubes filled with Dibromohexane (DBH) to the base of the flume and a syringe. As the sensors were very sensitive to pressure variations (pressure range from -250 to $250 \mathrm{~Pa}$ ), the syringe was essential for off-setting the sensor by an appropriate value; the sensors were calibrated for each run. The uncertainty of the pressure measurements was $\pm 3 \%$ according to the manufacturer. Tests were carried out in static conditions and with viscous flows to evaluate the performance of the system. Since authors ${ }^{11}$ recommended the use of screens (with a very fine mesh, typically $20 \mu \mathrm{m}$ square openings) placed across the holes to avoid disturbances due to the particles, we tried to optimize the pressure measurements. We observed no significant difference between screened and unscreened holes in our facility. Since DBH had a higher density than the particles and was characterized by low surface tension, particles did not penetrate into the tube and there was no pressure drop at the interface between DBH (filling the tube) and trimix (in the suspension flowing down the flume).

At time $t=0$, we released a fixed volume of suspension from a reservoir by raising a lockgate. The subsequent motion was recorded. Table I summarizes the three runs presented in this paper. We use the same nomenclature as for Paper I. ${ }^{61}$ We define a three-dimensional Cartesian coordinate system in which the $x$ axis points down the flume, the $y$ axis is in the direction of the upward pointing normal, and the $z$ axis is in the cross-stream direction The flume entrance is at $x=0$. The downstream and cross-stream velocity components are denoted by $u$ and $v$. The initial volume per unit width is $A$. The flow depth and front position are denoted by $h(x, t)$ and $x_{f}(t)$, respectively. 
TABLE I. Features of the different runs: mean solids fraction $\phi$, initial mass $m$, particle size distribution, the estimate of the bulk viscosity using the Krieger-Dougherty relation $\mu(\phi)=\mu_{f}\left(1-\phi / \phi_{m}\right)^{-\beta}$ (with $\beta=2$ and $\phi_{m}=0.625$ ), the characteristic flow depth $H_{*}$, the velocity and time scales $U_{*}=\rho g H_{*}^{2} \sin \theta / \mu(\phi)$ and $T_{*}=L_{*} / U_{*}$, and the flow Reynolds number $R e=\rho U_{*} H_{*} / \mu$. For all runs, the particle density, fluid density, fluid viscosity, and flume inclination were kept constant: $\rho_{f}$ $=\rho_{p}=1.184 \mathrm{~g} / \mathrm{cm}^{3}, \mu_{f}=0.124 \mathrm{~Pa} \mathrm{~s}, \theta=25^{\circ}$. The length scale is $L_{*}=2.55 \mathrm{~m}$ (the distance from the flume inlet to the main observation window). The flume length is $L=3 \mathrm{~m}$. The flow depth scale is $H_{*}=A / L_{*}$, where $A=m /(\rho W)$ denotes the initial volume per unit width (with $W=10 \mathrm{~cm}$ the flume width). The nomenclature is the same as that used in Paper I. ${ }^{61}$

\begin{tabular}{lcccccccc}
\hline \hline Run & $\phi$ & $m(\mathrm{~g})$ & Distribution & $\mu(\mathrm{Pa} \mathrm{s})$ & $H_{*}(\mathrm{~m})$ & $U_{*}(\mathrm{~m} / \mathrm{s})$ & $T_{*}(\mathrm{~s})$ & $R e$ \\
\hline B & 0.580 & 6000 & sieved & 23.9 & 0.020 & 0.081 & 31 & 0.080 \\
D & 0.595 & 6000 & sieved & 54 & 0.020 & 0.036 & 71 & 0.016 \\
I & 0.595 & 7940 & raw & 54 & 0.026 & 0.063 & 40 & 0.036 \\
\hline \hline
\end{tabular}

\section{EXPERIMENTAL RESULTS}

\section{A. Outline}

A wealth of different behaviors was observed depending on the mean solids fraction $\phi .{ }^{12}$ For $\phi \leq 0.57$, the suspension flowed like a viscous fluid. In particular, the front position varied with time as $t^{1 / 3}$, a scaling consistent with the theory of thin elongating Newtonian flows down a sloping bed. ${ }^{13}$ For $\phi \geq 0.61$, the flow came rapidly to rest. In the $0.56-0.61$ range, we observed three distinct regimes: at early times, the suspension moved downstream like a viscous avalanche in agreement with observations made, for instance, by Bonnoit et al. ${ }^{14}$ For this reason, we refer to this regime as the macro-viscous regime, studied in detail in Paper I. ${ }^{61}$ Before the front reached the end of the flume, the free surface became increasingly bumpy as fractures developed along the free surface. This second flow regime was thereafter called the fracture regime. These fractures marked the transition to a plastic regime, in which the suspension experienced repetitive stick-slip cycles and flowed downslope intermittently until it came to a final stop.

Figure 1(a) shows the variations of front position with time for two different solids fractions, $\phi=0.580$ (Run B) and $\phi=0.595$ (Run D). All other parameters were kept constant. For the sake of comparison, the data were nondimensionalized and plotted on a log-log diagram. At short times $(\hat{t}<0.1)$, the front position closely followed the theoretical solution $\hat{x}_{f}=(9 \hat{t} / 4)^{1 / 3}$ representing the evolution of the front position for a homogeneous Newtonian fluid ${ }^{13,15}$ (macro-viscous regime). At later times, there was a sudden transition to another regime, which was reflected by a kink in the $\hat{x}_{f}(\hat{t})$ curve. At $\hat{t} \sim 0.95$ for Run B, the $\hat{x}_{f}(\hat{t})$ curve flattened out, which meant that the front slowed down significantly. At $\hat{t} \sim 0.15$ for Run $\mathrm{D}$, the $\hat{x}_{f}(\hat{t})$ curve started to exhibit step-shaped variations. The suspension moved intermittently during episodes of slipping and stayed in an arrested state for varying lengths of time [see Fig. 1(b)]. The time between two episodes of slipping increased from less than $2 \mathrm{~s}$ to more than $90 \mathrm{~s}$. This stick-slip motion lasted for a very long time (up to $40 \mathrm{~min}$ ). Ultimately, separation between fluid and solid phases occurred as previously observed by Nsom. ${ }^{16}$

Our main finding was that the transition to the stick-slip regime was correlated with the bottom fluid pressure. Figure 2 shows the time variation of the flow depth along with the interstitial fluid pressure head $h_{p}=P_{f} /(\rho g \cos \theta)$, with $P_{f}$ the bottom fluid pressure, measured at $x=255 \mathrm{~cm}$. A striking feature of this figure is the position of the fluid pressure head relative to the flow depth. Until $t \sim 4 \mathrm{~min}$, the flow depth varied substantially due to the propagation of waves (fracture regime), but the pressure head exceeded the flow depth, which meant that the bottom pore pressure exceeded the total normal stress $\Sigma_{y y}=\rho g h \cos \theta$ so that the particle contribution to the stress tensor should be positive (tensile stress). At long times, $t>4 \mathrm{~min}$, the flow entered a plastic regime characterized by intermittent motion and oscillations of the basal fluid pressure (plastic regime). Once stick slip occurred, the pore pressure dropped below the normal stress $\Sigma_{y y}$ and showed fluctuations induced by the stick and slip phases. This meant that the particle stress changed sign and became negative (compressive stress). Although we failed to find a criterion for the transition from macro-viscous to fracture regimes, the transition from fracture to plastic regimes was clearly correlated with (perhaps 

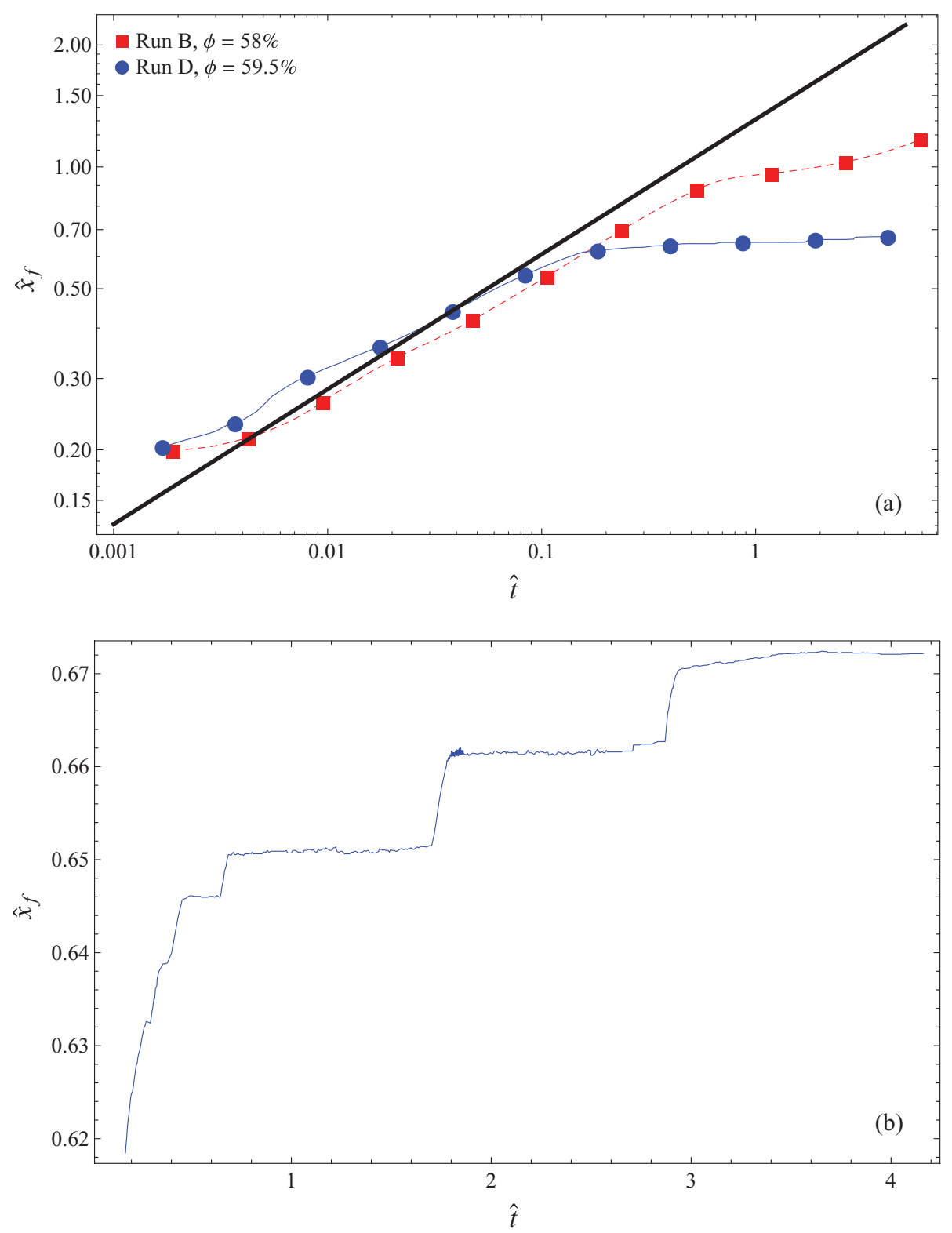

FIG. 1. (a) Position of the front as a function of time for Runs B and D. We also show the similarity solution $\hat{x}_{f}=(9 \hat{t} / 4)^{1 / 3}$. (b) Detail of the front position $\hat{x}_{f}(t)$ as a function of time for Run D. The released mass was $6 \mathrm{~kg}$ for both runs. The solids fraction was 0.580 (Run B) or 0.595 (Run D). For the sake of comparison, the data were scaled: $\hat{x}=x / L_{*}$ and $\hat{t}=t / T_{*}$ with the following scales: $L_{*}=2.55 \mathrm{~m}$ (distance from flume entrance to point of measurement), $T_{*}=L_{*} / U_{*}$, $U_{*}=\rho g H_{*}^{2} \sin \theta / \mu(\phi)$, and $H_{*} L_{*}=A$. We have also introduced $\mu(\phi)=\mu_{f}\left(1-\phi / \phi_{m}\right)^{-\beta}$ (Krieger-Dougherty's viscosity function) with $\beta=2$ and $\phi_{m}=0.625$ (maximum solids fraction).

dictated by) the sign of the excess pore pressure $P_{f}^{\prime}=P_{f}-\rho g h \cos \theta$, which was positive in the fracture regime, but negative in the plastic regime (see also Subsection 2 of the Appendix for a discussion of the interpretation of this behavior).

\section{B. Analysis of Run I}

The transition from the macro-viscous to the plastic regime was not smooth. An intermediate regime (fracture regime) took place between these two regimes. To better understand this transition 


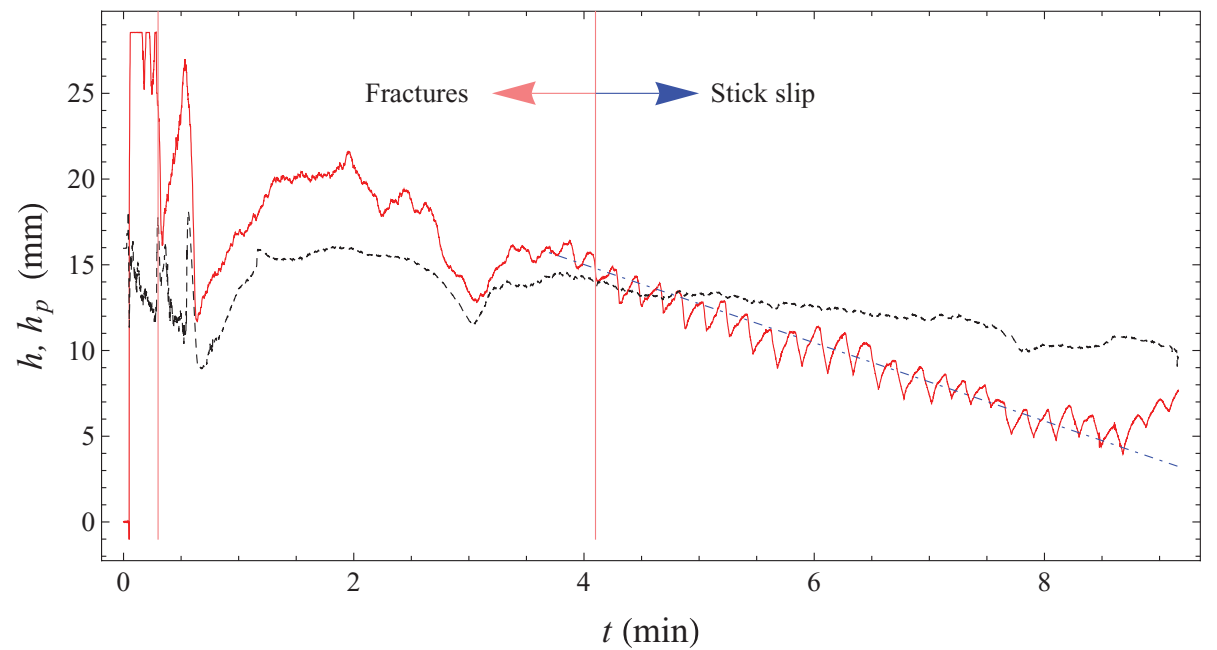

FIG. 2. Evolution of the flow depth $h$ (dashed line) and interstitial fluid pressure head $h_{p}=P_{f} /(\rho g \cos \theta)$ (solid line, red online). The dot-and-dash line (blue online) represents the pore pressure decrease attributed to pore pressure diffusion. Measurements were taken at $z=5 \mathrm{~cm}$ (centerline of the flume) and downstream coordinate $x=255 \mathrm{~cm}$.

from one regime to the next, we present the experimental data pertaining to each regime for Run I. Before describing the fracture regime (see Sec. III C) and the plastic regime (see Sec. III D), we will take a look at the time series of the depth-averaged velocity, flow depth, and bottom fluid pressure to get a better idea of how each regime is distinguished from the others.

Figure 2 shows the time variation of the flow depth along with the interstitial fluid pressure head $h_{p}=P_{f} /(\rho g \cos \theta)$, with $P_{f}$ the bottom fluid pressure, measured at $x=255 \mathrm{~cm}$, while Fig. 3 shows the time evolution of the depth-averaged velocity, measured at the centerline. At short times $(t<30 \mathrm{~s})$, the velocity was "high" (typically $3 \mathrm{~cm} / \mathrm{s}$ ), as the leading edge of the flow passed in front of the observation window. The flow depth decreased slowly, but continuously from $16 \mathrm{~mm}$ to $12 \mathrm{~mm}$.

Later, two fractures occurred at times $t=30 \mathrm{~s}$ and $t=48 \mathrm{~s}$. Prior to fracture, the velocity briefly increased, corresponding to the upper layer sliding at high speed on the bottom layer. Once the upper layer had been completely removed, the velocity strongly decreased. After the second fracture, the velocity fell below $3 \mathrm{~mm} / \mathrm{s}$ and was almost constant for $3 \mathrm{~min}$. During this time the flow slid on the bottom of the channel (with shear confined to a thin layer along the flume bottom) until it first came to rest, corresponding to the beginning of the stick-slip regime ( $t=3.8 \mathrm{~min})$. The flow depth was roughly uniform along the flume. The velocity profiles across the stream showed that all the shearing was concentrated within a thin layer along the flume bottom. Note that after $t>1 \mathrm{~min}$, the depth-averaged velocity was fairly constant and did not depend on the flow depth. For instance, at $t \sim 2 \mathrm{~min}$, the flow depth rose from $12 \mathrm{~mm}$ to $21 \mathrm{~mm}$ without causing any increase in the depth-averaged velocity.

The occurrence of intermittent motion was a robust feature of all the neutrally-buoyant particle suspensions we tested: changing the size distribution (by sieving the particles) or changing the interstitial fluid composition (while keeping the density-matched character) did not alter the flow pattern. We repeated the experiments, keeping the same protocol, without noticing any significant variation except that the time to reach the stick-slip regime could vary a great deal from one run to another with the same conditions. We also changed the boundary conditions slightly (e.g., wetting the flume bottom with Dibromohexane). Again, we did not notice any significant qualitative change in behavior.

Another distinctive feature of the behavior of particle suspensions in the high-concentration limit was the absence of shear within the bulk, except in the close vicinity of the front and along the flume bottom. Typically, for $\phi=0.595$, the depth-averaged velocity was $\sim 10 \mathrm{~mm} / \mathrm{s}$, the flow depth was $\sim 20 \mathrm{~mm}$ (i.e., 200 particle diameters), and the thickness of the basal shear layer was $\sim 500 \mu \mathrm{m}$ ( 3 particle diameters). Figure 4 shows the evolution of the basal pore pressure $P_{f}$ and 

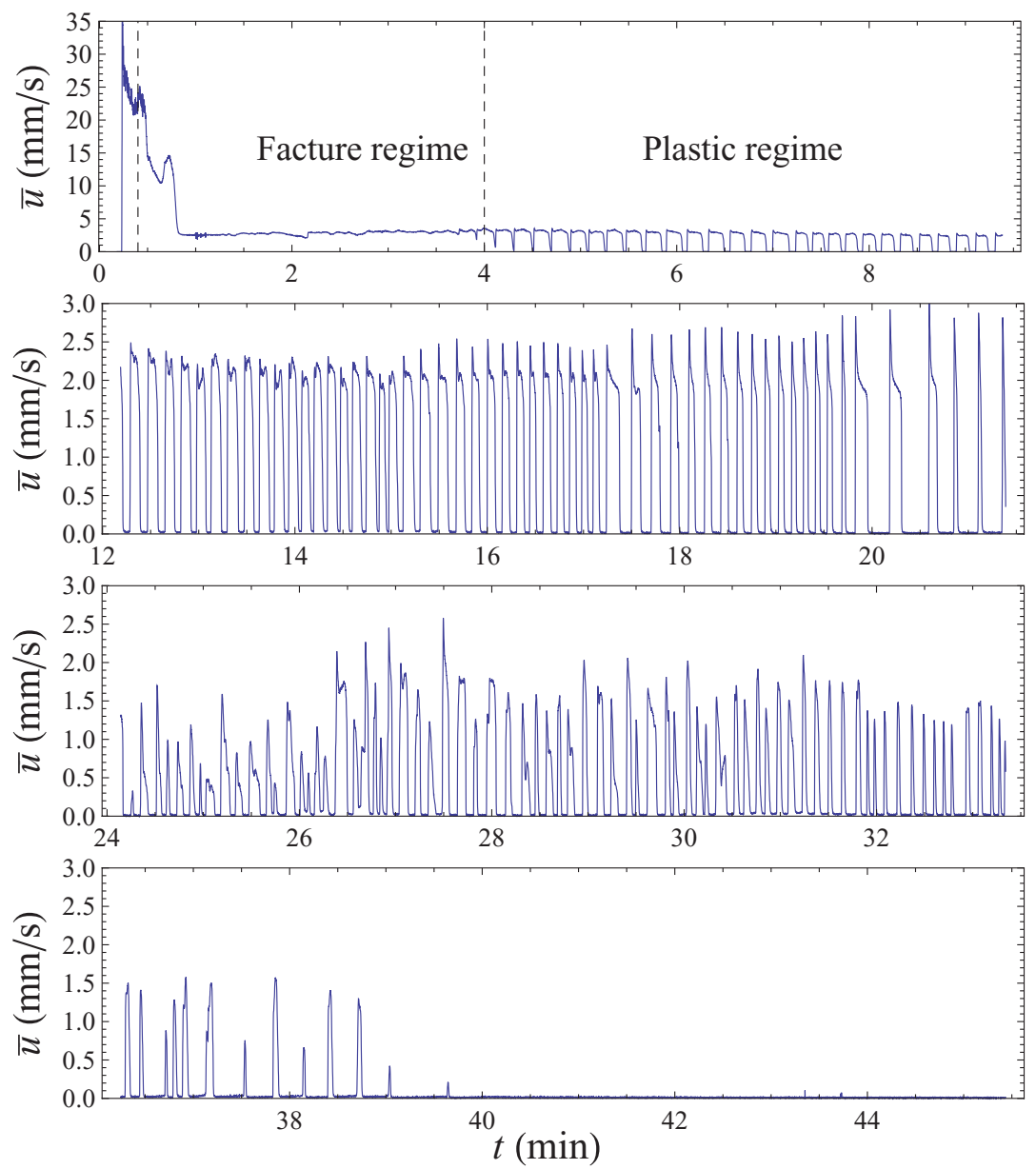

FIG. 3. Evolution of the depth-averaged velocity $\bar{u}$ for Run I. Measurements were taken at $z=5 \mathrm{~cm}$ (centerline of the flume) and downstream coordinate $x=255 \mathrm{~cm}$. Every $9 \mathrm{~min}$, we had to clear the cache memory of the camera, an operation that lasted 3 min and during which we could not take measurements. This explains why there was no velocity record for $9 \leq t \leq 12 \mathrm{~min}, 21 \leq t \leq 24 \mathrm{~min}$, and $33 \leq t \leq 36 \mathrm{~min}$.
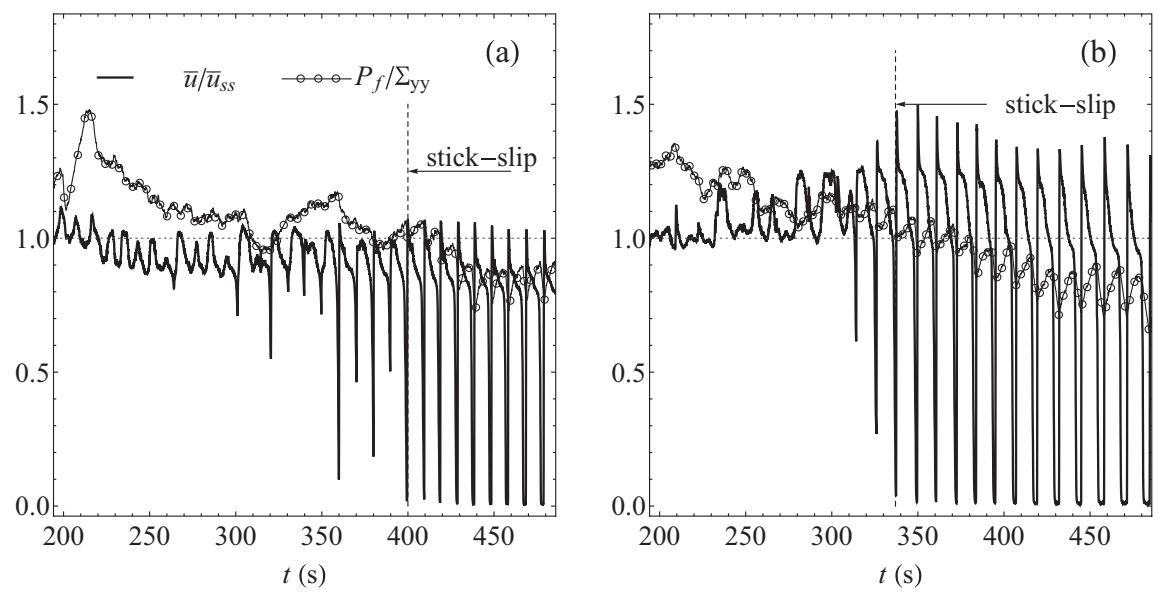

FIG. 4. Time variations in the ratios $P_{f} / \Sigma_{y y}$ and $\bar{u} / \bar{u}_{s s}$ for two realizations of Run I. Measurements (bottom fluid pressure and flow depth) taken at $x=255 \mathrm{~cm}$. The normal stress $\Sigma_{y y}$ was computed as $\Sigma_{y y}=\rho g h \cos \theta$. The steady state (timeaveraged) velocities were $\bar{u}_{s s}=3.15 \mathrm{~mm} / \mathrm{s}$ (a) and $\bar{u}_{s s}=2.72 \mathrm{~mm} / \mathrm{s}$ (b). The stick-slip regime occurred at $t=398 \mathrm{~s}$ (a) and at $t=337 \mathrm{~s}(\mathrm{~b})$. 
the depth-averaged streamwise velocity $\bar{u}$ for two realizations of Run I. Prior to the first halt, the velocity experienced a few cyclic oscillations, with increasing amplitude. At time $t=398 \mathrm{~s}$ for Run $\mathrm{I} / \mathrm{a}$ and $t=337 \mathrm{~s}$ for Run $\mathrm{I} / \mathrm{b}$, the suspension came to a brief halt. It then accelerated vigorously. Although the runs were not identical [in particular, the oscillations were more pronounced for Run $\mathrm{I} / \mathrm{b}$ and the first-halt times differed significantly], the pattern was the same.

\section{Fracture regime}

When the solids fraction was sufficiently high $(\phi \geq 0.575)$, the body of the avalanche started fracturing like a "landsliding" mass of soil. Although this regime was primarily distinguished by the propagation of bumps and ripples along the free surface, it had nothing to do with free-surface instabilities (roll waves), which are observed at much higher Reynolds numbers. ${ }^{17}$

Here we focus on Run I, but the same kind of behavior was observed for other runs. A few seconds after the release (typically $t>3-5 \mathrm{~s}$ for a mass of $\sim 8 \mathrm{~kg}$ ), we observed that the free surface deformed and became bumpy. As shown by Figure 5, the fractures formed a regular threedimensional pattern (with an initial wavelength of approximately $10 \mathrm{~cm}$, growing to $100 \mathrm{~cm}$ ) which spanned the entire length of the flow. The fractures looked like those observed in cohesive soils, i.e., with a curved slip surface inside the material, along which the shear was localized. When these fracture regions grew sufficiently in size, they modified the local velocity field substantially. Despite the fractures, the general shape of the body was still well predicted using lubrication theory (see Paper I). ${ }^{61}$

Figure 6 shows a sequence of snapshots taken every $0.5 \mathrm{~s}$. At $t=49 \mathrm{~s}$ (the first image in the upper left corner of the figure), the suspension moved almost as a rigid block since there was virtually no shear in the vertical direction (all the shear being localized within a thin layer along the solid boundary). At $t=50 \mathrm{~s}$, there was a significant decrease in the flow depth, accompanied by a marked reduction in the velocity. Interestingly, at $t=50.5 \mathrm{~s}$, this region quickly collapsed and gave rise to two flow regions separated by a 3-mm thick layer, inclined at $25^{\circ}$ to $30^{\circ}$ with respect to the bottom and characterized by a sharp velocity gradient: the upper region clearly slid on the lower region, which slid on the flume bottom. At $t=52 \mathrm{~s}$ (image in the lower left corner of the figure), the fractured region left the observing window and a calmer region (low velocity, no shear) occupied the field filmed by our camera; note that the flow depth eventually started increasing again due to subsequent fractures passing across the observation window. Figure 7 reports the cross-stream flow-depth profiles at different times. The profiles were symmetric and exhibited two cusps, which were the lateral borders of the fracture (as shown by Figure 5, the slipping surface was delineated or flanked by two curvilinear levees along each sidewall). The fracture process lasted a few tens of

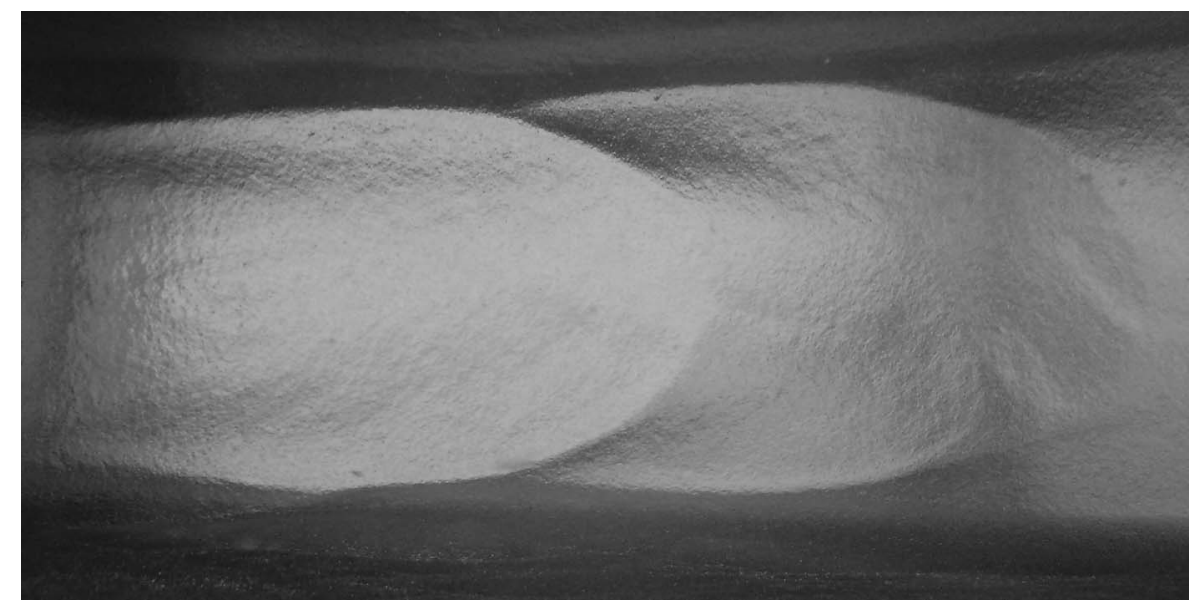

FIG. 5. Picture of a fracture viewed from the top. The flume width is $10 \mathrm{~cm}$, the typical length of the fracture is $10-15 \mathrm{~cm}$ (flow from right to left). 

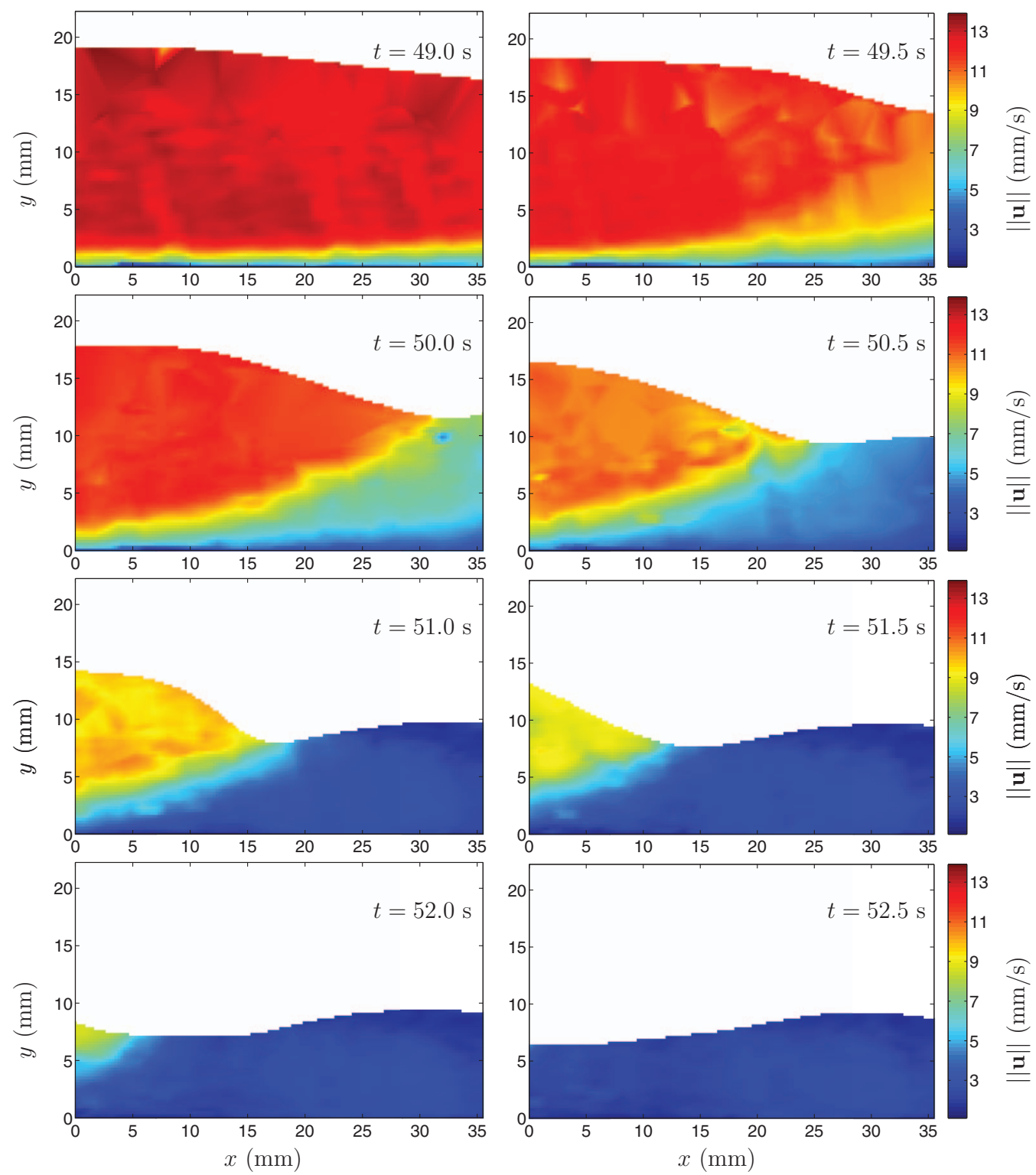

FIG. 6. Snapshots showing the velocity field within the flowing suspension during the fracture process (flow from right to left) for Run I. We show only the velocity norm $\|u\|=\sqrt{u^{2}+v^{2}}$. The time increment between successive images was $0.5 \mathrm{~s}$. Measurements were made at $x=255 \mathrm{~cm}$ along the centerline of the flume $(z=5 \mathrm{~cm})$.

seconds. Its effects became progressively weaker, as shown at $t=100 \mathrm{~s}$ for Run I, where the flow was a thin creeping layer (approximately $13 \mathrm{~mm}$ in thickness, moving at a mean velocity of $3 \mathrm{~mm} / \mathrm{s}$ ).

\section{Plastic regime}

At longer times ( $t>4 \mathrm{~min}$ for Run I), the plastic regime occurred. It was characterized by intermittent motion (with phases during which the suspension accelerated sharply and reached a quasi-steady regime, and phases during which the suspension came to a sudden halt) and, ultimately, by complete stoppage of the suspension.

As shown by Fig. 3, the duration of the slip phases decreased with time. At $t=4$ min (start of the regime), the typical duration was $11 \mathrm{~s}$ while at $t=32 \mathrm{~min}$, the typical duration fell to less than $3 \mathrm{~s}$. In contrast, the duration of the stick phases increased more markedly, from a few milliseconds 

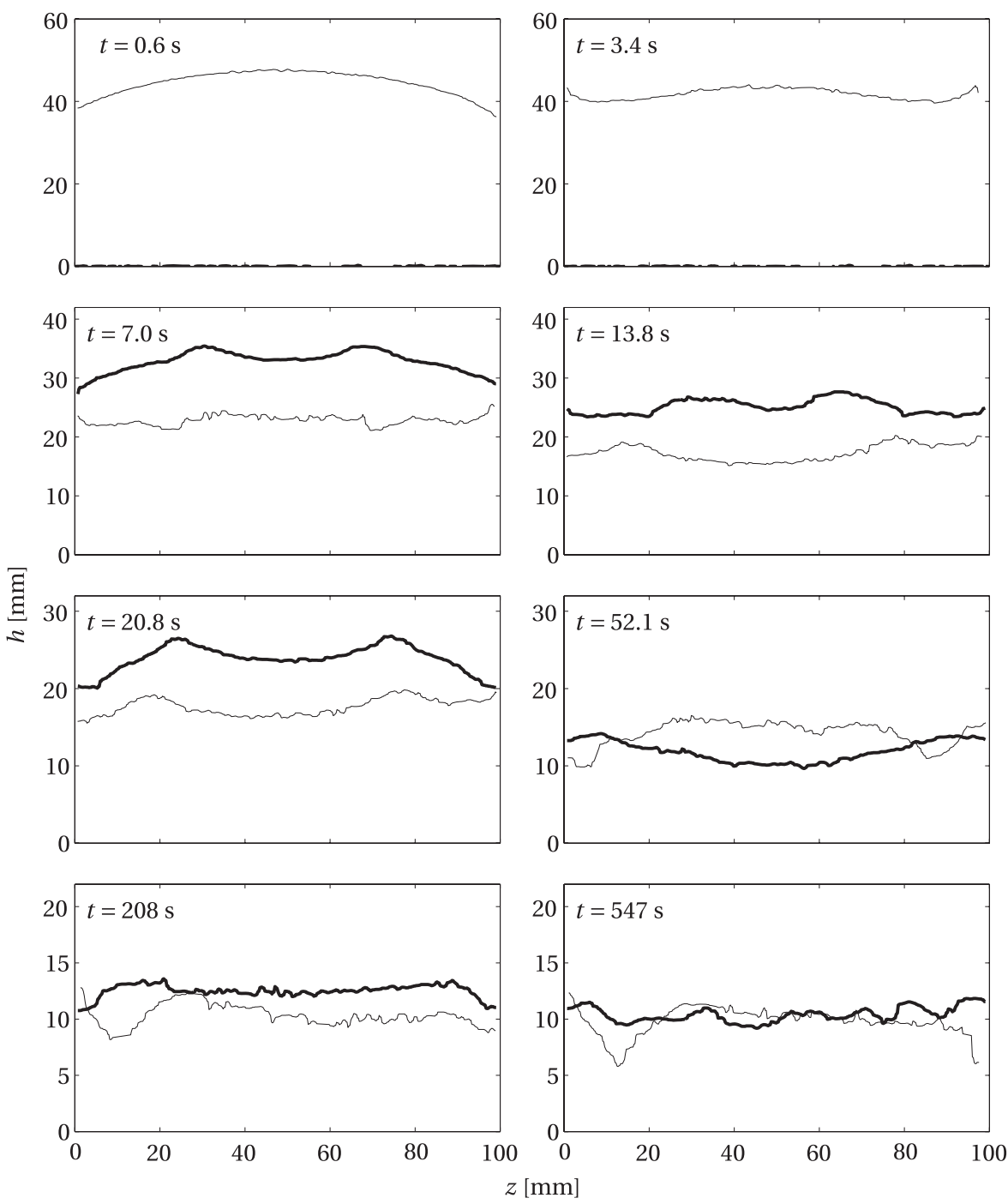

FIG. 7. Cross-stream flow depth profiles for Run I. They were taken at different times and two different positions along the channel $x=66 \mathrm{~cm}$ (thin line) and $x=205 \mathrm{~cm}$ (thick line).

(at $t=4 \mathrm{~min}$ ) to $12 \mathrm{~s}$ (at $t=32 \mathrm{~min}$ ). The stick-slip regime lasted a few tens of minutes until the material came progressively to a final halt. Complete standstill was first observed in the upper part of the flume and it took several minutes for all of the suspension to come to an arrested state. At the end of the stick-slip regime a 10-mm thick deposit of suspension covered the bottom. Fluid seepage (self-filtration) was then observed: the interstitial fluid was drained from the bulk and flowed in narrow rivulets, which progressively incised the bulk down to the base (see Fig. 8). Interestingly, this phase separation was fairly fast since it occurred a couple of hours after the start of the experiment, whereas in the sedimentation experiments we carried out during our preliminary tests, we observed no phase separation even after one week.

While measuring the velocity field $\mathbf{u}=(u(x, y, z, t), v(x, y, z, t))$ in the central plane and at $x$ $=255 \mathrm{~cm}$, we also monitored the evolution of the interstitial fluid pressure at the bottom (basal pore pressure) $P_{f}$ and the flow depth $h$. Figure 9 shows the time variations in $P_{f}, h$ and the depth-averaged streamwise and cross-stream components of the velocity ( $\bar{u}$ and $\bar{v}$, respectively) during one cycle of slipping. Figure 10 is a close-up of the former figure to show the evolution at short times after the start of a slip phase. As soon as the suspension started to move, the normal velocity component $v$ increased significantly for approximately $0.2 \mathrm{~s}$, showing that the material underwent dilatancy. Near 

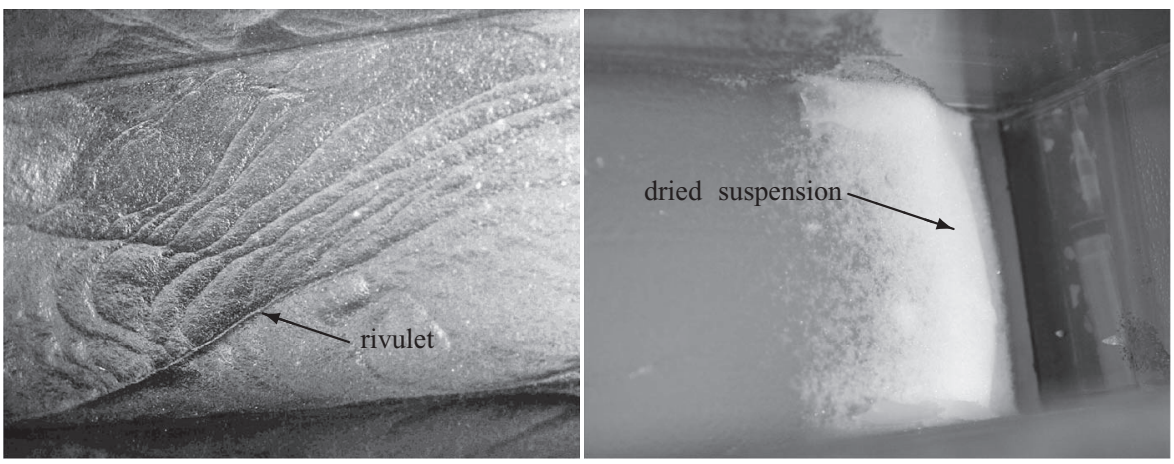

FIG. 8. Picture of the free surface taken $60 \mathrm{~min}$ after release for Run I. Left: picture taken at $x \sim 150 \mathrm{~cm}$. At the bottom of the image a rivulet draining the interstitial fluid can be identified. Other ripples are formed by the slow creeping of the bulk (flow from right to left). Right: a view of the upper end of the reservoir, fluid seepage has dried the bulk and only the solid frame remains at rest (flow from right to left).

the bottom of the flume the vertical velocity $v$ remained small, whereas close to the free surface, in the upper layers of the flow, $v$ reached its maximal value. Dilatancy was, however, small: the maximal vertical velocity $v$ was $0.2 \mathrm{~mm} / \mathrm{s}$ near the free surface and lasted less than $0.4 \mathrm{~s}$, producing a maximum displacement of 80-100 $\mu \mathrm{m}$ (i.e., almost a particle diameter). $v$ became negative $0.4 \mathrm{~s}$ after the motion started $(t=1.4 \mathrm{~s})$, meaning that the flow was contracting. After $2 \mathrm{~s}$, the initial flow height was recovered. Therefore, surprisingly, contraction of the flow was not correlated with flow arrest. The maximal streamwise velocity $\bar{u}$ was reached at the same time as the maximal dilatation (i.e., just before $v$ became negative). Then, $\bar{u}$ slowly decreased to an almost constant value. Up until $t=2 \mathrm{~s}$, values of $u(x, y, z, t)$ near the bottom were $7 \%$ smaller than values obtained close to the free surface, meaning that the flow was slightly sheared during the acceleration of the bulk. After $t \sim 2 \mathrm{~s}$, the values of $u(x, y, z, t)$ near the bottom of the flume became identical to the free surface velocity: the bulk was slipping with no internal deformation. This is particularly obvious in Fig. 10, in which cross-stream velocity profiles $u(x, y, z, t)$ computed at different times are shown. Profiles (a) to (d) correspond to acceleration, while profiles (e) to (i) correspond to deceleration.

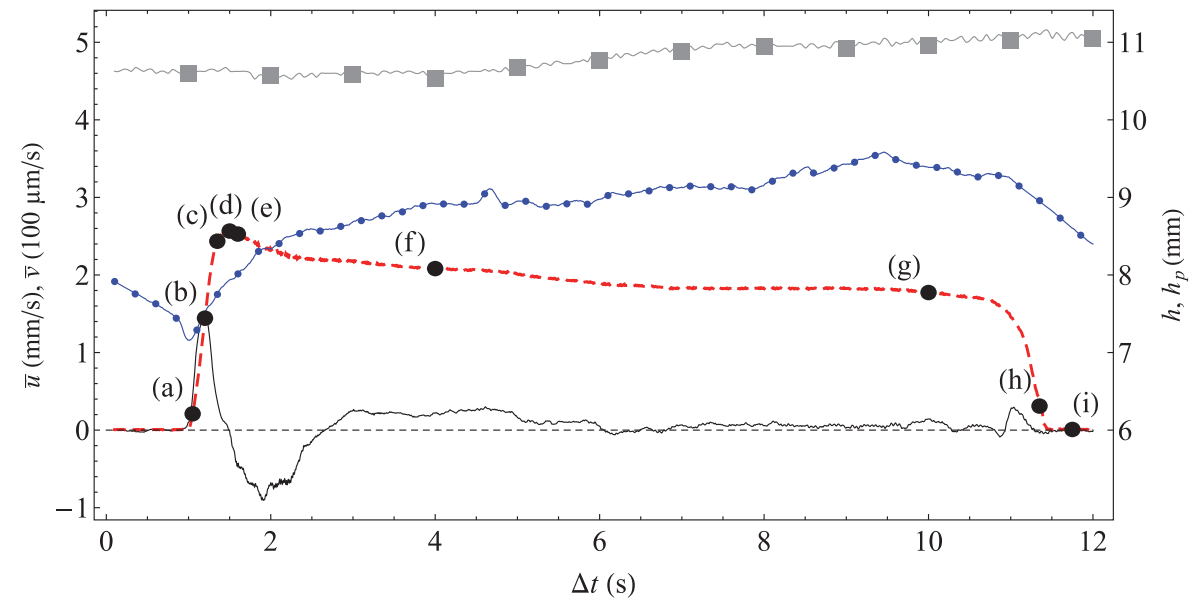

FIG. 9. Time variations in the flow variables over one typical phase of slipping: the dashed (red online) curve represents the depth-averaged streamwise velocity $\bar{u}$ while the thin solid line is the normal component $\bar{v}$. The (blue online) curve marked with dots is the basal fluid pressure head $h_{p}$ while the curve flagged with gray squares is the flow depth. Dots labeled from (a) to (i) refer to the times at which the velocity profiles of Figure 10 have been plotted: (a) $\Delta t=1.05 \mathrm{~s}$, (b) $\Delta t=1.20 \mathrm{~s}$, (c) $\Delta t=1.35 \mathrm{~s}$, (d) $\Delta t=1.50 \mathrm{~s}$, (e) $\Delta t=1.60 \mathrm{~s}$, (f) $\Delta t=4.00 \mathrm{~s}$, (g) $\Delta t=10 \mathrm{~s}$, (h) $\Delta t=11.35 \mathrm{~s}$, and (i) $\Delta t=11.75 \mathrm{~s}$. Time $\Delta t=0$ corresponds to $623 \mathrm{~s}$ after the initial release. Measurements were taken on Run I at the centerline $(z=5 \mathrm{~cm})$ at downstream coordinate $x=255 \mathrm{~cm}$. 


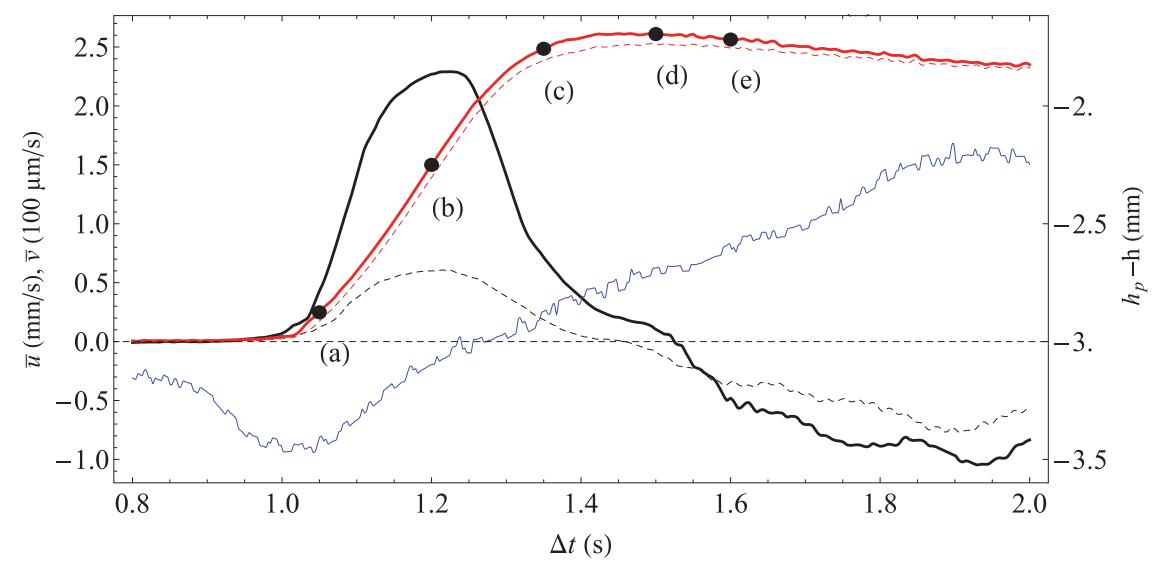

FIG. 10. Detail of Fig. 9. We split the depth-averaged velocities in two: bottom region ( $y=0$ to $y=h / 2)$ and top region ( $y$ $=h / 2$ to $y=h$ ). Dashed lines show the bottom region whereas the solid line shows the top region; the thick (red online) line represents the streamwise velocity $u$. The thin (black online) line is the normal velocity component $v$. We also plotted the excess pore pressure head. The dots indicate the times at which the $u(x, y, z, t)$ velocities were taken. Time $\Delta t=0$ corresponds to $623 \mathrm{~s}$ after the initial release.

Pore pressure began to increase at the same time as the flow started to move, as shown in Fig. 10. The magnitudes of the basal pore pressure fluctuations were much larger than the flow depth variations, meaning that the pore pressure was not hydrostatic. Excess pore pressures $\left(P_{f}^{\prime}\right.$ $\left.=P_{f}-\rho g h \cos \theta\right)$ remained negative during the entire stick-slip cycle ranging from $-3.5 \mathrm{~mm}$ of fluid just before the flow initiation to $-1.5 \mathrm{~mm}$ of fluid at $t=10 \mathrm{~s}$. Once the maximal pore pressure was reached, the pressure started to relax at first slowly, then more rapidly. This decay induced deceleration of the suspension and eventually flow arrest. At rest the pressure continued to decrease linearly until the next slip cycle (Fig. 11, lower panel).
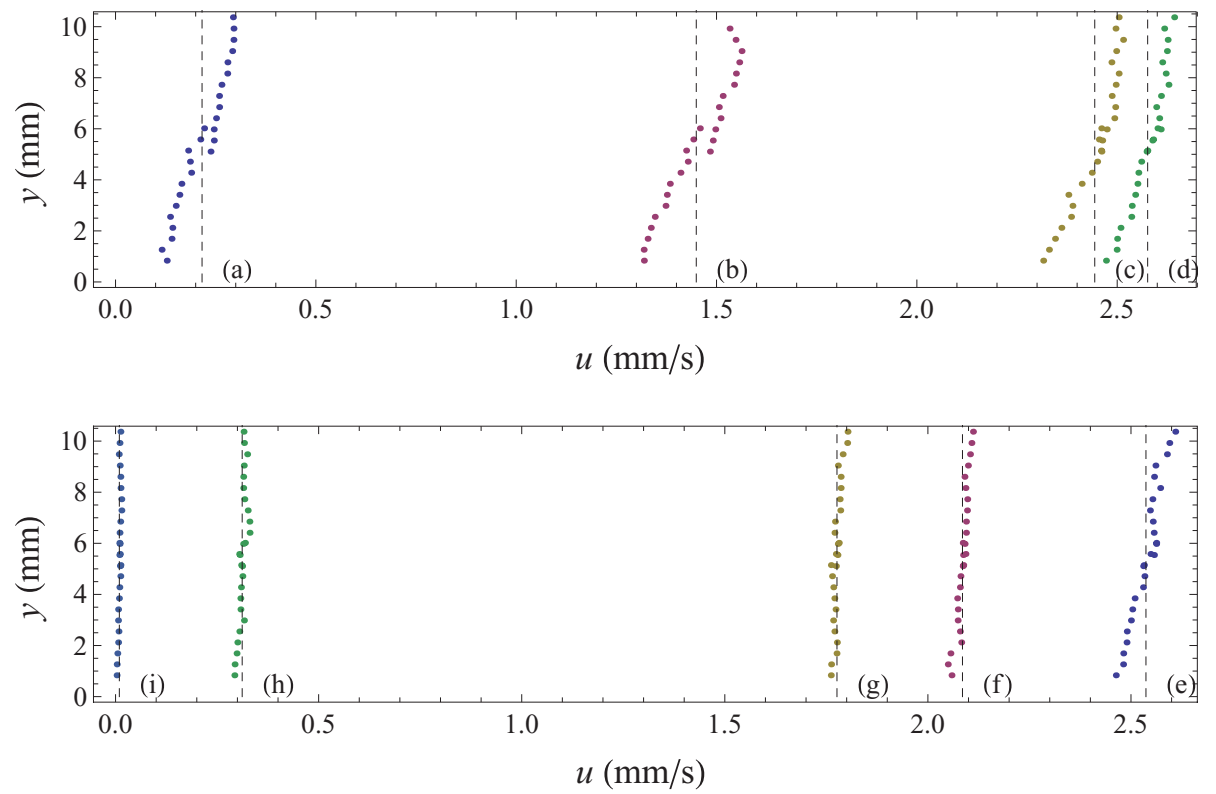

FIG. 11. Velocity profiles $u(x, y, z, t)$ at times (upper panel) (a) $\Delta t=1.05 \mathrm{~s}$; (b) $\Delta t=1.20 \mathrm{~s}$, (c) $\Delta t=1.35 \mathrm{~s}$, (d) $\Delta t=1.50 \mathrm{~s}$, (lower panel) (e) $\Delta t=1.60 \mathrm{~s}$, (f) $\Delta t=4.00 \mathrm{~s}$, (g) $\Delta t=10 \mathrm{~s}$, (h) $\Delta t=11.35 \mathrm{~s}$, and (i) $\Delta t=11.75 \mathrm{~s}$. Time $t=0$ corresponds to $623 \mathrm{~s}$ after the initial release. The vertical dashed lines represent the mean (depth-averaged) velocities. Measurements were taken on run $\mathrm{I}$ at the centerline $(z=5 \mathrm{~cm})$ and downstream coordinate $x=255 \mathrm{~cm}$. 


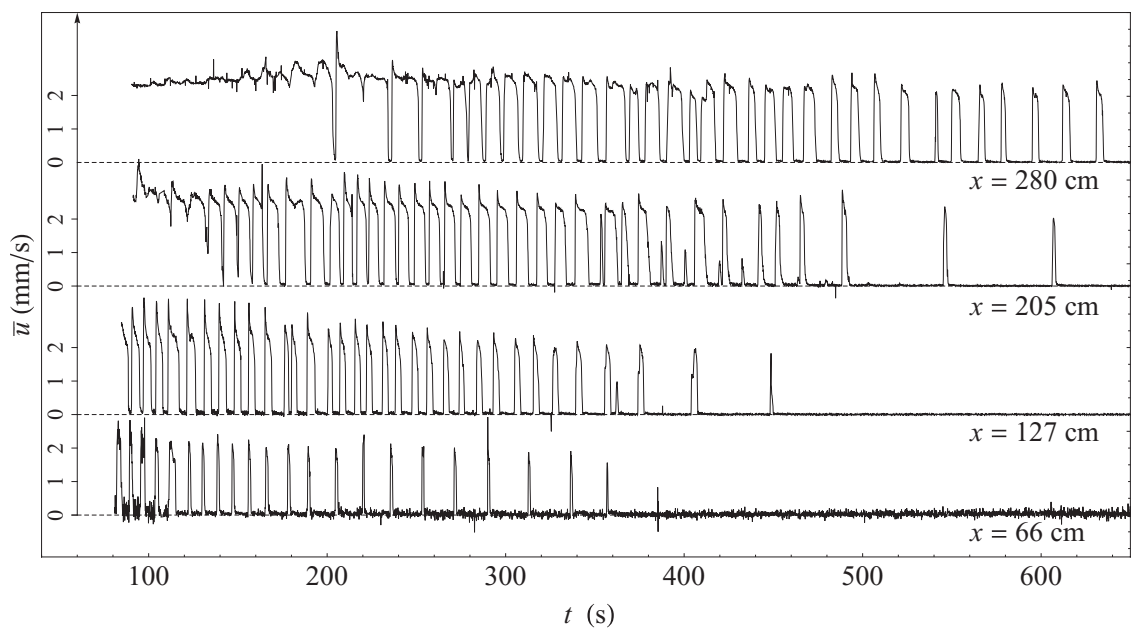

FIG. 12. Time evolution of the free-surface velocities for Run I. The velocities were taken simultaneously at different places along the centerline of the channel $x=66 \mathrm{~cm}, 127 \mathrm{~cm}, 205 \mathrm{~cm}$, and $280 \mathrm{~cm}$.

Initially, we thought of cyclic traveling waves as originating from the flume inlet and causing the pore pressure fluctuations. However, cross-correlating the velocity signals recorded in different places $(x=66,127,205$, and $280 \mathrm{~cm})$ did not reveal any traveling waves at the channel length scale. As shown by Fig. 12, the slipping phases occurred at different times and were not interrelated. However, by focusing on smaller length scales ( $L \sim 60 \mathrm{~cm}$ or less) as shown by Fig. 13, we observed pressure waves. Limited propagation lengths may have been caused by a frequency mismatch: at a given place along the channel, as illustrated by Fig. 3, the shape of the stick-slip cycle changed with time, the duration of the stick phase increased whereas the slip phase duration decreased. Due to the fact that the stick-slip regime did not start at the same time everywhere along the channel, but was initiated earlier in the region adjacent to the door before propagating downstream, at a time $t$ the stick-slip frequency varied at different places along the channel. Therefore, synchronized movement was impossible. The pressure wave velocity $(c \sim 40 \pm 5 \mathrm{~cm} / \mathrm{s})$ was almost constant over

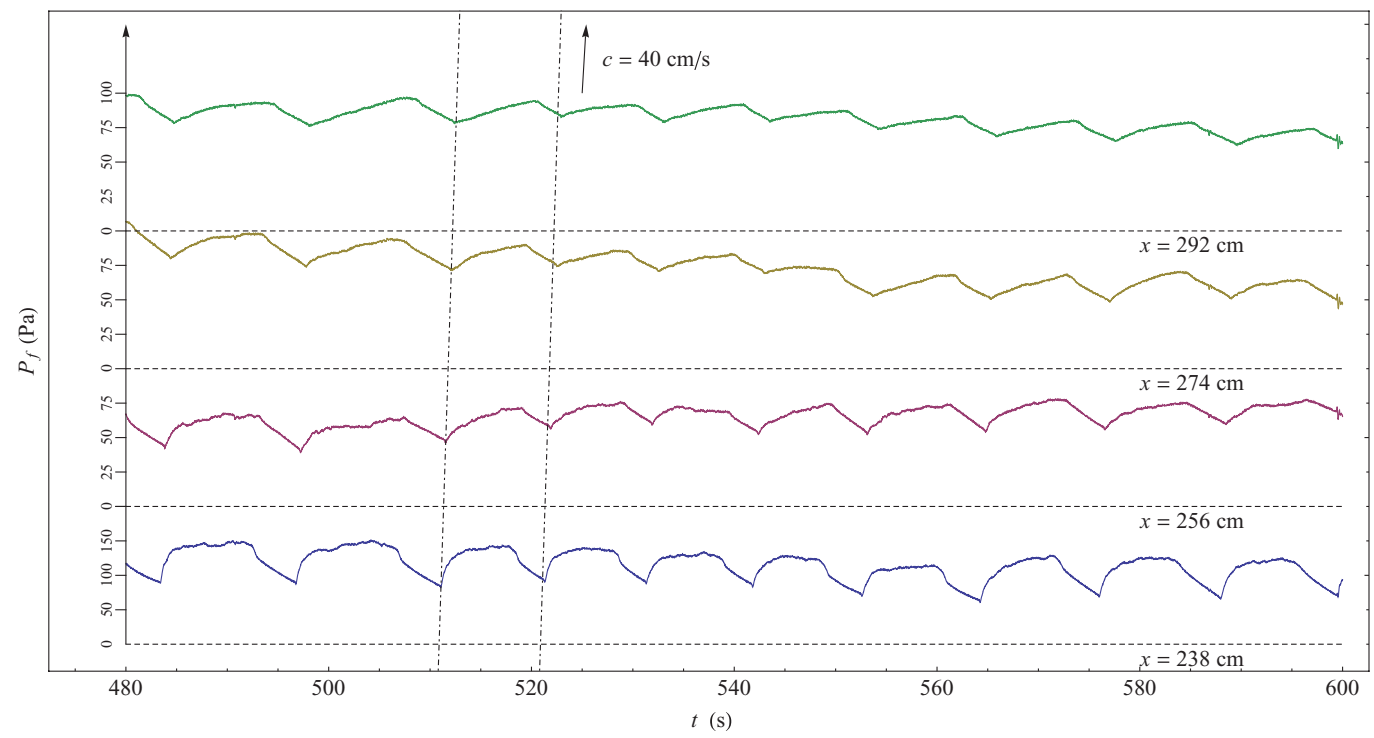

FIG. 13. Details of the bottom pore pressure recorded along the channel at four different places for Run I: $x=238.3 \mathrm{~cm}$, $256.4 \mathrm{~cm}, 274.2 \mathrm{~cm}$, and $292.3 \mathrm{~cm}$. The vertical dot-and-dash lines show the propagation of pressure waves with a celerity of $c=40 \pm 5 \mathrm{~cm} / \mathrm{s}$. 
time. It was slightly higher than the gravity wave velocity $(\sqrt{g h} \sim 30 \mathrm{~cm} / \mathrm{s})$. Note also that the typical duration of a slipping phase (about $10 \mathrm{~s}$ ) corresponds to the time taken by a pressure wave to go back and forth over the entire flume length $(t \sim 2 L / c \sim 15 \mathrm{~s})$. The mean bottom pressure at the upstream station $(x=238.3 \mathrm{~cm})$ was systematically higher than that at the downstream station $(x=292.3 \mathrm{~cm})$, which meant that there was a pressure gradient in the streamwise direction, which was as high as $200 \mathrm{~Pa} \mathrm{~m}^{-1}$ just after the flow entered a plastic regime $(240<t<300 \mathrm{~s})$, but dropped to $16 \mathrm{~Pa} \mathrm{~m}^{-1}$ at long times. The amplitudes of the pore pressure fluctuations were also more pronounced for the upstream station than for the downstream station.

\section{INTERPRETATION}

\section{A. Analysis and comparison with similar works}

Intermittent motion in a flume was observed by Iverson et al. ${ }^{18}$ who investigated the raininduced triggering of landslides by running large-scale experiments. Their experiments consisted of a layer of loose soil at rest on a sloping bed, in which the water content was progressively increased with the aid of sprinklers and drains. Depending on its initial porosity, the soil layer partially liquified or moved intermittently. Iverson ${ }^{19}$ developed a constitutive equation combining Coulomb friction, contraction/dilatancy, and generation/diffusion of pore pressure. Idealizing the soil layer as a sliding block with velocity-dependent friction, Schaeffer and Iverson ${ }^{20}$ derived a simplified set of governing equations that admitted analytical solutions. In particular, they showed that timeperiodic behavior appeared in the form of intermittent motion as the pore pressure fluctuated due to pore contraction/expansion and diffusion of pore pressure throughout the bulk. This theoretical description is clearly in line with our observations, yet, agreement is not complete. In the paper by Schaeffer and Iverson, ${ }^{20}$ a lot of idealization was necessary to make the equations tractable. In particular, the authors assumed the Coulomb friction coefficient to be a decreasing function of the velocity (this assumption was required to explain why the steady state solution was unstable), and the pore pressure to obey an advection diffusion equation (even though fluid pressure diffusion originates from fluid compressibility, a process that is unlikely to be significant for shallow layers of soil). In this case and for certain initial conditions, their governing equations lost stability through a subcritical Hopf bifurcation. In the phase plane showing the evolution of the velocity and excess pore pressure, the solution experienced sticking behavior during which the pressure increased and slipping behavior during which the pressure was mainly decreasing. In their model, the duration of the stick phase increased and trajectories tended to a periodic orbit. In our case, although we observed an increasing duration of the stick phases (see Fig. 3), the pore pressure decreased during these stick phases and the trajectories in the $\bar{u}-P_{f}^{\prime}$ phase plane were counterclockwise (see Fig. 14).

Pailha, Nicolas, and Pouliquen ${ }^{21}$ experimentally investigated the flow initiation of granular materials fully immersed in a liquid. To that purpose, they suddenly tilted a box filled with liquid containing the setup. Then they monitored the basal pore pressure and the velocity of the solid phase. They found that for sufficiently high solids fractions $(\phi \sim 0.58)$, the flow did not start immediately when the box was inclined. A delay was observed during which the flow was creeping and basal pore pressure fell. Once the flow developed fully, the pressure increased again to a steady state value. Using the idealized picture of landslides described by Iverson, ${ }^{19}$ Pailha, Nicolas, and Pouliquen ${ }^{21}$ showed that this delay was due to the time necessary for the fluid to diffuse through the granular layers. Although our experiments also provided evidence for pore pressure diffusion throughout the bulk, they differed from those conducted in latter work in that the motion was intermittent and there was no creeping motion prior to a sudden acceleration of the mass at rest.

Kulkarni, Metzger, and Morris ${ }^{22}$ investigated gravity- and pressure-driven flows of polystyrene suspensions in a channel whose cross-section narrowed abruptly. They observed that for solids concentrations exceeding 0.58 , flow alternated periodically between fast and slow motions if the pressure gradient applied to the suspension was sufficiently large. As there was a small density difference between the carrier fluid and the particles (about 1\%-2\%), they suggested that a percolating network of particles in close contact was formed under the action of the piston pushing the suspension. 


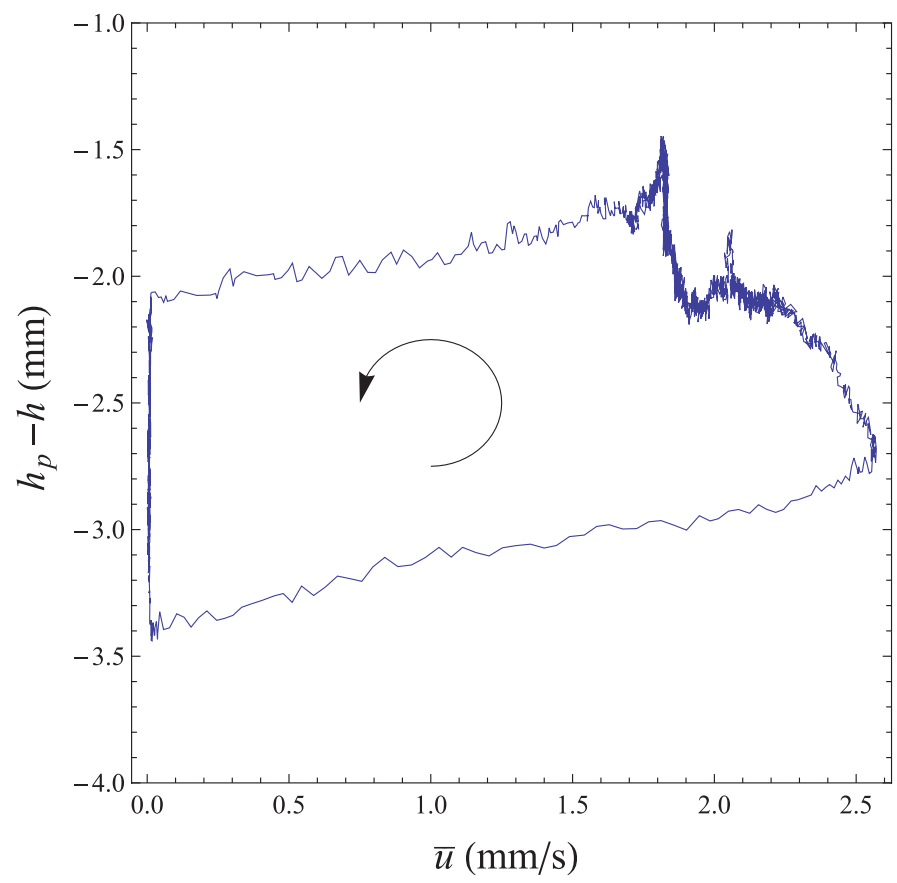

FIG. 14. A stick-slip cycle: the excess pore pressure head $h_{p}-h=P_{f} /(\rho g \cos \theta)-h$ as a function of the streamwise depth-averaged velocity $\bar{u}$, the trajectory is counterclockwise. We have extracted a single cycle (Run I).

This network created strong resistance to motion, but when it broke, this resistance decreased suddenly, causing significant acceleration of the flow. Because of the narrowing of the crosssection, new networks formed again promptly. Our experimental data exhibits similarities with those findings, but as the density difference between the solid and fluid phases was vanishingly small in our experiments and there was no geometrical constraint on the flow, the physical process governing stick-slip motion might be different.

\section{B. Jamming as an explanation of stick-slip motion}

In light of previous experiments and interpretations, we propose the following explanation of stick-slip motion. Shortly after the release, the flow acceleration is sufficient to shear the suspension, especially within the leading edge (the curvature of the free surface may even promote this shear) and along the flume bottom. This explains why the front behavior is similar to that of a homogeneous Newtonian fluid (see Fig. 1). This induces an increase in the pore fluid pressure as a result of compaction (consistently with the interpretation given by geomechanicians) and/or the momentum balance between the fluid and solid phases when the bulk is vigorously sheared. This is in accordance with the pore pressure record at $x=255 \mathrm{~cm}$ (see Fig. 2). As long as the pore pressure $P_{f}$ exceeds the vertical normal stress $\Sigma_{y y}$, the particle contribution to the total stress $\left(\Sigma_{y y}^{p}=P_{f}-\Sigma_{y y}\right)$ is positive, which means that this component of the particle stress tensor is a tension rather than a pressure.

With time, the excess pore pressure diffuses slowly throughout the bulk. In the mechanics of porous media composed of a rigid skeleton, the diffusion timescale is negligibly small for incompressible interstitial fluids, ${ }^{23}$ but when the medium is composed of loose grains, authors ${ }^{19,24,25}$ have suggested that bulk compressibility gives rise to pore pressure diffusion even though the interstitial fluid is incompressible, as is obvious in Fig. 2. This decrease in pore pressure is slow, but faster than the decrease in the flow depth. At a finite time after the release ( $t \sim 4 \mathrm{~min}$ on Fig. 2$)$, the pore pressure drops below the total normal stress $\Sigma_{y y}$, which implies that the particle stress $\Sigma_{y y}^{p}$ becomes negative (compressive). 
The sudden change in the sign of $\Sigma_{y y}^{p}$ may explain why the particles jam by forming a network of particles resisting the gravitational forces. This explanation is, however, incomplete as it does not describe how the network forms and then breaks. Because the suspension is perfectly densitymatched, the particles are unlikely to interact through direct frictional contacts, nor form arches that would span the flow depth or the width, as is the case in dry granular flows. ${ }^{26}$ An externally applied force is thus needed to ensure stability of the percolating network. One, and perhaps the sole, possibility is the capillary force arising from the interfacial tension between the interstitial fluid and air (see Subsection 3 of the Appendix). As suggested by a number of authors, ${ }^{9,27-30}$ the protrusions of the surface caused by the particles (of radius $a$ ) give rises to a jump in the normal stress $\Sigma_{y y}^{p}$ proportional to $\gamma / a$ and a jump in the pore pressure that scales as $-\gamma / a$. For our suspensions, the order of magnitude of the stress jump is $30 \mathrm{~Pa}$, to be compared with the bottom shear stress $\Sigma_{x y}=$ $\rho g h \sin \theta=50 \mathrm{~Pa}$.

The typical size of the particle network must also compare with the typical flow depth if it is to block the flow. Very little is known about the cluster size for non-buoyant particle suspensions in the high Péclet number limit (i.e., for non-Brownian particles). For this reason, it is difficult to reason beyond simple speculation. Jenkins et al. ${ }^{31}$ ran experiments, which showed that at moderate Péclet numbers, the mean cluster size was about 5 particles and the maximum observed size was 70 particles. For non-buoyant non-Brownian particle suspensions, Bonnoit et al. ${ }^{32}$ showed that there was a critical flow depth $\xi \approx 0.2\left[\mu^{2}(\phi) /\left(\rho^{2} g\right)\right]^{1 / 3}$ associated with a major change in the bulk viscosity, presumably related to particle cluster formation. For Run I, we found $\xi \sim 12 \mathrm{~mm}$, a value reasonably close to the critical depth $h \sim 14 \mathrm{~mm}$ (or, equivalently, 130 particle diameters), which marks the transition to the plastic regime in Fig. 2. This result supports the idea that below a critical flow depth, particle networks are able to span the entire depth and, together with capillary forces, to increase flow resistance.

When these particle networks are sufficiently strong to resist the gravitational forces, the flow comes to a halt. Meanwhile, the interstitial fluid continues to flow through the pores of the jammed particles. This is process is known as self-filtration. ${ }^{33}$ According to the Darcy law

$$
\boldsymbol{u}_{f}=-\frac{k}{1-\phi} \frac{1}{\mu_{f}} \nabla P_{f}^{\prime}
$$

with permeability $k=4 \times 10^{-12} \mathrm{~m}^{2}$, fluid seepage induces a pressure gradient in the streamwise direction. Very low seepage velocities are able to generate significant pressure gradients. For instance, a velocity of $u_{f} \sim 0.02 \mu \mathrm{m} / \mathrm{s}$ creates a pressure gradient $\Delta P_{f}^{\prime} / \Delta x \sim 200 \mathrm{~Pa} \mathrm{~m}^{-1}$, consistent with our pore pressure records (see Fig. 13).

Because we see no precursor phenomenon (such as an increase in the pore pressure) prior to each phase of slipping, we assume-as Kulkarni, Metzger, and Morris ${ }^{22}$ did-that the jammed particles are in an unstable state ${ }^{34}$ and that the particle cluster may break at any time. Once this breaking begins, the suspension dilates and the pore pressure increases, which leads to a substantial decrease in shear resistance, a process that is consistent with the physical picture of wet granular materials described by Iverson ${ }^{24}$ and Pailha, Nicolas, and Pouliquen. ${ }^{21}$ This causes the suspension to accelerate sharply. The maximum velocity is reached when the suspension is maximally dilated (see Fig. 10). Once this maximum velocity his reached, the suspension compacts again, but in contrast with the theory of wet granular flow, this compaction is not followed by a pore pressure reduction. On the contrary, $P_{f}$ continues to increase for a few seconds. When it decreases again, the velocity drops rapidly and the flow comes to a new halt. Interestingly, there is no critical value of the shear stress that would correspond to the initiation of slipping. Indeed, the value of the pore pressure at the start of the slip phase decreases with time whereas the flow depth is slightly decreasing, implying that the stress ratio $K=\Sigma_{x y} / \Sigma_{y y}^{p}$ decreases with time without affecting the occurrence of slip and/or reaching a constant value. It is thus impossible to determine a fixed value for the friction coefficient $K$, which would be related to a Coulomb yield stress.

Although we can qualitatively explain many of our observations, the origin of the alternation between stick and slip phases is more difficult to grasp. We fail to understand a number of features, including the nearly constant duration of slip phases, the pressure waves moving at constant velocity, 
the relationship between pore pressure and velocity in the slip phase, and why pore pressure diffused throughout the bulk.

\section{CONCLUSION}

Within the framework of fluid mechanics, fluid pressure is not usually expected to play a part in the dynamics of free-surface flows as it does not feature in the fundamental part of the constitutive equation (the deviatoric stress tensor). In contrast, fluid pressure (pore pressure) plays a key role in shear strength in the soil-mechanics theory of granular materials. When pore pressure exceeds the total normal stress, the granular material is in a liquified state whereas in the opposite case, the particles experience frictional contacts. Moreover, excess pore pressure (defined as pore pressure minus normal stress) is related to the motion of the fluid phase relative to the solid skeleton (Darcy's seepage process). In this paper, we provided evidence that during its early stages, flow was characterized by (positive) excess pore pressure, whereas at long times, the basal pore pressure was lower than the vertical normal stress. Furthermore, at short times, the ore pressure was well-correlated with the depth variations whereas at long times, this correlation vanished.

We conclude that even in the absence of a density difference between the fluid and the particles, concentrated suspensions may exhibit plastic behavior when the pore-fluid pressure is insufficient to maintain the particles in a fluidized state. Although our results seem to highlight the emergence of a Coulomb yield stress as a result of the drop in pore pressure, they are not in full agreement with soil plasticity theory. Among others, there is no critical shear stress that controls the start of the slip phases as we would have expected, had there been a well-defined friction coefficient. Moreover, the relation between pore pressure, dilatancy, and rate of deformation differs from the physical picture drawn by Iverson. ${ }^{24}$ The alternative explanation, jamming, seems more viable. In agreement with the findings of Bonnoit et al., ${ }^{32}$ we think that there may be a critical flow depth below which particle clusters are sufficiently large to span the entire depth. These clusters transmit the capillary forces arising from the interfacial tension between the interstitial fluid and air, which may force the particles to jam and block the flow. Self-filtration (fluid seepage) makes the jammed clusters unstable and whenever the latter break, the suspension begins to flow again.

\section{ACKNOWLEDGMENTS}

The work presented here was supported by the Swiss National Science Foundation under Grant No. 200021-105193/1 (a project called "Transient free-surface flows of concentrated suspensions Application to geophysical flows," funded by an R'Equip grant), the competence center in Mobile Information and Communication Systems (a center supported by the Swiss National Science Foundation under Grant No. 5005-67322, MICS project), the competence center in Environmental Sciences (TRAMM and APUNCH projects), and specific funds provided by EPFL (vice-présidence à la recherche). We are grateful to Belinda Bates for copyediting the paper.

\section{APPENDIX: THEORY}

The goal of this appendix is to see how the concept of fluid pressure is introduced for a particle suspension regarded as a continuum. We start with microstructural theories, which infer the bulk constitutive equation by averaging the local mass and momentum balance equations (see Subsection 1 of this Appendix). In such theories, the interstitial fluid pressure is a variable that reflects fluid incompressibility. It is determined solely by solving the full governing equations with the appropriate boundary conditions. More recently, other theories have been proposed, which regard particle suspensions as saturated granular materials rather than fluids. Fluid pressure is then introduced by — or related to - empirical relations such as the Darcy equation (see Subsection 2 of this Appendix). In Subsection 3 of this Appendix, we see how surface tension induces a normal stress. 


\section{Microstructural theory}

The suspension is composed of non-colloidal particles within a Newtonian carrier fluid of viscosity $\mu_{f}$ and surface tension $\gamma$. Both solid and fluid phases have the same density $\rho_{f}=\rho_{p}$ $=\rho$. The particle concentration is denoted by $\phi$ and its average throughout the bulk is called the (mean) solids fraction $\bar{\phi}$. The particles are assumed to be spheres of radius $a$. Assuming that the Reynolds number is small at any scale (from particle to flow scales), we can derive the averaged mass and momentum balance equations for either phase by averaging the local mass and momentum equations (Cauchy equations) using a statistical average $\langle\cdot\rangle$. The derivation of the equations is now well-established and we refer the interested reader to the key literature. ${ }^{35-41}$ For the sake of completeness and to make the comparison with Subsection 2 of this Appendix, we repeat the main points here. For the microstructural analysis, we need to distinguish between local and phaseaveraged velocities. Upper-case letters refer to phase averaged quantities: $\boldsymbol{U}_{p}=\left(U_{p}, V_{p}\right)$ is the local velocity averaged over the solid phase while $\boldsymbol{U}_{f}=\left(U_{f}, V_{f}\right)$ is the velocity averaged over the fluid phase. Likewise, the local stress tensor is denoted by $\sigma$, and the solid- and fluid-phase-averaged stress tensors are $\boldsymbol{\Sigma}_{p}$ and $\boldsymbol{\Sigma}_{f}$. The normal stresses are denoted by $\Sigma_{x x}$ and $\Sigma_{y y}$ while the shear stress is $\Sigma_{x y}$.

We take advantage of the fact that the density is constant throughout the bulk and that there is no slip at the fluid-particle interface. The averaged mass balance equations are then

$$
\begin{array}{r}
\partial_{t} \phi+\nabla \cdot\left(\phi \boldsymbol{U}_{p}\right)=0, \\
\partial_{t}(1-\phi)+\nabla \cdot\left[(1-\phi) \boldsymbol{U}_{f}\right]=0,
\end{array}
$$

in which $\boldsymbol{U}_{p}=\left\langle\chi_{p} \boldsymbol{u}\right\rangle / \phi, \boldsymbol{U}_{f}=\left\langle\chi_{f} \boldsymbol{u}\right\rangle /(1-\phi), \chi_{k}(k=p$ or $f)$ is the phase indicator function (e.g., $\chi_{f}(\boldsymbol{x})=1$ if $\boldsymbol{x}$ lies within the fluid phase), and $\phi=\left\langle\chi_{p}\right\rangle$. Other forms of these equations are in common use. First, by summing (A1) and (A2), we obtain $\nabla \cdot \boldsymbol{U}=0$, where the bulk velocity is denoted by $\boldsymbol{U}=\phi \boldsymbol{U}_{p}+(1-\phi) \boldsymbol{U}_{f}$. As both phases are incompressible, the bulk is also incompressible. The second variant is obtained by using (A1) together with $\nabla \cdot(\phi \boldsymbol{U})=\boldsymbol{U} \cdot \nabla \phi$ to obtain

$$
\frac{\partial \phi}{\partial t}+\boldsymbol{U} \cdot \nabla \phi=-\nabla \cdot \boldsymbol{J},
$$

with $\boldsymbol{J}=\phi\left(\boldsymbol{U}_{p}-\boldsymbol{U}\right)$ the particle flux relative to the bulk motion. This equation is one of the fundamental equations that underpin suspension balance theory. ${ }^{40}$

In the absence of inertial terms, the averaged momentum balance equations read

$$
\begin{gathered}
\rho \phi \boldsymbol{g}+\nabla \cdot\left\langle\chi_{p} \boldsymbol{\sigma}_{p}\right\rangle+\left\langle\delta_{i} \boldsymbol{\sigma}_{f} \cdot \boldsymbol{n}\right\rangle=0, \\
\rho(1-\phi) \boldsymbol{g}+\nabla \cdot\left\langle\chi_{f} \boldsymbol{\sigma}_{f}\right\rangle-\left\langle\delta_{i} \boldsymbol{\sigma}_{f} \cdot \boldsymbol{n}\right\rangle=0,
\end{gathered}
$$

where $\sigma_{p}$ and $\sigma_{f}$ denote the particle and fluid stress tensors, $\boldsymbol{n}$ is the outward-oriented normal to the particle interface, and $\delta_{i}$ is the interface indicator function. As the interstitial fluid is Newtonian, we can express $\boldsymbol{\sigma}_{f}$ as the sum of a pressure term and deviatoric contribution: $\boldsymbol{\sigma}_{f}=-p \boldsymbol{I}+\boldsymbol{d}_{f}$ with $\boldsymbol{I}$ the identity tensor, $\boldsymbol{d}_{f}=2 \mu \boldsymbol{e}_{f}$ the viscous stress deviator, and $\boldsymbol{e}_{f}=\frac{1}{2}\left(\nabla \boldsymbol{u}_{f}+\nabla \boldsymbol{u}_{f}^{\dagger}\right)$ the local rate of strain. For each equation (A4) and (A5), the first term on the left-hand side denotes the body force, the second term is the divergence of the averaged local stress tensor, while the third contribution represents the average forces exerted at the interfaces between the fluid and solid phases. This last term is often seen as the key contribution, which reflects the coupling between the two phases. Equations (A4) and (A5) can then be recast in the following form: ${ }^{42}$

$$
\begin{gathered}
\nabla \cdot\left\langle\chi_{p} \boldsymbol{\sigma}_{p}\right\rangle+\left\langle\delta_{i} \boldsymbol{d}_{f} \cdot \boldsymbol{n}\right\rangle=0, \\
\rho \boldsymbol{g}-\nabla P_{f}+\nabla \cdot\left\langle\chi_{f} \boldsymbol{d}_{f}\right\rangle-\left\langle\delta_{i} \boldsymbol{d}_{f} \cdot \boldsymbol{n}\right\rangle=0
\end{gathered}
$$

with $P_{f}=\left\langle\chi_{f} p_{f}\right\rangle /(1-\phi)$ the mean pressure. To close the equations, we need to compute the particle and fluid stress tensors as well as the interface force $\left\langle\delta_{i} \boldsymbol{d}_{f} \cdot \boldsymbol{n}\right\rangle$. While the fluid stress tensor can be 
expressed analytically,

$$
\boldsymbol{\Sigma}_{f}=-P_{f} \boldsymbol{I}+\left\langle\chi_{f} \boldsymbol{d}_{f}\right\rangle=-P_{f} \boldsymbol{I}+2 \mu_{f} \boldsymbol{E},
$$

where $\boldsymbol{E}=\frac{1}{2}\left(\nabla \boldsymbol{U}+\nabla \boldsymbol{U}^{\dagger}\right)$ denotes the averaged rate of strain, the two other contributions cannot be determined explicitly. Furthermore, the local stress tensor is not defined inside the particles as they are rigid. The averaged particle stress tensor is then defined as

$$
\boldsymbol{\Sigma}_{p}=\left\langle\chi_{p} \boldsymbol{\sigma}_{p}\right\rangle=a\left\langle\delta_{i} \boldsymbol{n}\left(\boldsymbol{\sigma}_{p} \cdot \boldsymbol{n}\right)\right\rangle
$$

In general, estimations of this tensor have been obtained using different approaches. In one approach, the starting point is the structure of the stress tensor in the small-concentration limit. The effect of the solids fraction is to enhance the bulk viscosities and develop non-isotropic normal stresses. ${ }^{43-45}$ The particle stress tensor is written

$$
\boldsymbol{\Sigma}_{p}=-\mu_{f} \eta_{n}(\phi) \dot{\gamma} \boldsymbol{Q}+2 \mu_{f}\left(\eta_{s}(\phi)-1\right) \boldsymbol{E}
$$

where $\boldsymbol{Q}$ is a diagonal matrix with positive entries, $\dot{\gamma}=(2 \boldsymbol{E}: \boldsymbol{E})^{1 / 2}$ the second invariant of the strainrate tensor, and $\eta_{s}(\phi)$ and $\eta_{n}(\phi)$ are two viscosity coefficients. The model parameters $\left(\boldsymbol{Q}, \eta_{n}, \eta_{s}\right)$ are calibred from experiments. This formulation of the constitutive equation receives considerable experimental support. ${ }^{40,45,46}$

In another approach, the essence is that the particle stresses are generated at the particle level by strong interactions between particles. ${ }^{47-50}$ In its simplest form, this approach regards the divergence of the bulk viscosity function as the consequence of the squeezing forces between particles, whose amplitudes vary as $1 / \zeta$, where $\zeta$ denotes the face-to-face distance between particles. More elaborate approaches require the tensor description of microstructure emphasizing the role of the particle arrangement in the stress generation, and use representation theorems to infer the constitutive equation. ${ }^{42,51,52}$

The momentum balance equation for the bulk is obtained by summing Eqs. (A6) and (A7). We end up with a conservation equation of that is remarkably simple

$$
\rho \boldsymbol{g}+\nabla \cdot \boldsymbol{\Sigma}=0 \text { with } \boldsymbol{\Sigma}=-P_{f} \boldsymbol{I}+2 \mu_{f} \boldsymbol{E}+\boldsymbol{\Sigma}_{p}
$$

with $\boldsymbol{\Sigma}_{p}$ given by Eq. (A10) or an alternative form.

A few remarks can be made about the microstructural theory before we apply it to our configuration. First, the interface force is often approximated by $\left\langle\delta_{i} \boldsymbol{\sigma}_{f} \cdot \boldsymbol{n}\right\rangle=-6 \pi \mu a f^{-1}(\phi)\left(\boldsymbol{u}_{p}-\boldsymbol{u}\right)$, with $f$ the hindrance sedimentation function. ${ }^{43,44}$ Using this closure equation, the momentum balance equation (A6) and (A3), in which $\boldsymbol{J}$ is replaced with $2 a^{2} f(\phi) \nabla \cdot \boldsymbol{\Sigma}_{p} /\left(9 \mu_{f}\right)$, we obtain a governing equation for $\phi$. Second, the fluid pressure has no specific role. The fluid being incompressible, its role is to ensure the consistency of the governing equation. It is to be determined in the solution of the governing equations. In the following, we will see how $P_{f}$ accommodates changes in the normal stress $\Sigma_{y y}$.

New insights are made possible by computing the stress state in either phase. For flow conditions close to simple shear (like the experiments presented in the body of this article), making use of the normal stress distribution $\Sigma_{y y}=\rho g(h-y) \cos \theta$ together with the definition of the stress tensors (A8) and (A10), we deduce that the normal and shear stresses are

$$
\Sigma_{y y}=-P_{f}-\mu_{f} \eta_{n}(\phi) \lambda_{2} \dot{\gamma}+\mu_{f} \eta_{s}(\phi) \partial_{y} V=-\rho g(h-y) \cos \theta \text { and } \Sigma_{x y}=\mu(\phi) \partial_{y} U
$$

with $\dot{\gamma} \approx\left(\left(\partial_{y} U\right)^{2}+2\left(\partial_{y} V\right)\right)^{1 / 2}$ and $\lambda_{2} \approx 0.6-0.8$ a constant. ${ }^{44,45}$ In limit of vanishing $V$ (genuinely simple-shear flow), all contributions to the normal stress are negative and the interstitial fluid pressure 
accommodates the particle stress changes

$$
P_{f}=\rho g(h-y) \cos \theta-\mu_{f} \eta_{n}(\phi) \lambda_{2} \dot{\gamma}=\left(1-\lambda_{2} \tan \theta \frac{\eta_{n}(\phi)}{\eta_{s}(\phi)}\right) \rho g(h-y) \cos \theta .
$$

This shows that except for the cases where $\theta=0$ or $\phi=0$, the fluid pressure departs from the hydrostatic pressure distribution and is slightly lower than the total normal stress (with the usual expressions and values, we have $\left.\lambda_{2} \tan \theta \eta_{n}(\phi) / \eta_{s}(\phi)<1\right)$. If we now consider the case $\partial_{y} V=O\left(\partial_{y} U\right)$ (a situation that arises in the vicinity of the front), then the pressure may exceed the total normal stress

$$
P_{f}=\left(1-\lambda_{2} \tan \theta \frac{\eta_{n}(\phi)}{\eta_{s}(\phi)}\right) \rho g(h-y) \cos \theta+\mu_{f} \eta_{s}(\phi) \partial_{y} V .
$$

This result also shows that the particle stress is positive. An excess interstitial fluid pressure has no special meaning in fluid mechanics, but it is of particular significance in soil mechanics as we will see in Subsection 3 of this Appendix.

\section{Insights from granular flow theory}

In Sec. I, there has been a bias in our approach: we have regarded highly concentrated suspensions as fluids with a high solids content. The other way around is to see these suspensions as granular materials whose pores are filled with fluid. Granular suspensions inherit some properties from granular materials. First, the particles are so close together that they may interact through contact forces, which gives rise to shear-thickening, jamming and networks of particles in close contact. ${ }^{53}$ When the particles are sufficiently rough or they are negatively buoyant, close (frictional) contact is possible between particles, which leads to a radical change in the bulk behavior (Coulomb like behavior). Closely packed granular assemblies are prone to dilatancy, that is, a shear-induced change of volume that causes a reorganization of the particle arrangement compatible with shear.

The nature of contact (lubricated or frictional) can be predetermined using a dimensionless number, sometimes referred to as the Leighton number, which is nothing but the ratio of viscous to buoyancy forces: ${ }^{54-56} L e \propto \mu_{f} \dot{\gamma} /(\Delta \rho g h)$ with $\Delta \rho$ the density mismatch between the fluid and solid phases. With this in mind, there is no possibility of frictional contact between smooth particles when $\Delta \rho=0$. This does not, however, remove the possibility of percolating networks that carry forces across long distances through lubricated contacts. On rare occasions in viscometric experiments, it is possible to impose the particle normal stress $\Sigma_{p}^{y y}$ experienced by the particles even though they are neutrally buoyant. An example is provided by Boyer, Guazzelli, and Pouliquen, ${ }^{57}$ who showed how the macro-viscous and granular rheologies were made compatible when using a proper parametrization. They conducted simple-shear experiments with neutrally buoyant particles in an annular shear cell. Introducing a dimensionless group $I_{v}=\mu_{f} \dot{\gamma} / \Sigma_{p}^{y y}$ (called the viscous number), they showed that the shear stress can equally be written $\Sigma_{p}^{x y}=\mu_{f} \eta_{s}(\phi) \dot{\gamma}$ or $\Sigma_{p}^{x y}=K\left(I_{v}\right) \Sigma_{p}^{y y}$ with $K$ an effective friction coefficient reminiscent of the Coulomb friction. They also demonstrated that in a steady state, the mean solids fraction was a function of the viscous number $\bar{\phi}=\phi_{m} /\left(1+\sqrt{I_{v}}\right)$. Note that in their experiments, the interstitial fluid pressure $P_{f}$ is left undetermined, which makes the rheological model more difficult to apply to situations in which the particle stress $\Sigma_{p}^{y y}$ is not imposed externally, but varies with the fluid pressure throughout the bulk.

Recently, Iverson ${ }^{24}$ and George and Iverson ${ }^{25}$ have developed a constitutive model, which is rooted more in soil mechanics than in suspension rheology. A dilatant frictional material follows a generalized Coulomb law: $\Sigma_{p}^{x y}=K \Sigma_{p}^{y y}$ where $K=\tan (\varphi+\psi)$ is the bulk friction coefficient, $\varphi$ the particle friction angle (a material constant), and $\psi$ is the shear-induced dilatancy angle (which varies with the shear rate and the solids fraction). These authors added an empirical equation that describes the coupling between the fluid pressure $P_{f}$, the particle stress $\Sigma_{p}^{y y}$, and the dilatancy in a simple shear flow:

$$
\nabla \cdot \boldsymbol{U}_{p}=\dot{\gamma} \tan \psi-\alpha \frac{d \Sigma_{p}^{y y}}{d t}
$$


where $\alpha$ is the mixture compressibility and $d \cdot / d t$ the Lagrangian derivative. As the bulk is incompressible, $\nabla \cdot \boldsymbol{U}_{p}=-\nabla \cdot \boldsymbol{J}$ with $\boldsymbol{J}=(1-\phi)\left(\boldsymbol{U}_{f}-\boldsymbol{U}_{p}\right)$ the seepage velocity. The Darcy law provides

$$
\boldsymbol{J}=-\frac{k}{\mu_{f}}\left(\nabla P_{f}-\rho \boldsymbol{g}\right),
$$

with $k$ the permeability, which is related to the particle size distribution and solids fraction. ${ }^{58}$ Note that in the original model, Iverson ${ }^{24}$ introduced the excess pore-fluid pressure $P_{f}^{\prime}=P_{f}-\rho g(h$ $-y) \cos \theta$. By combining Eqs. (A15) and (A16), we end up with a parabolic equation with a forcing term for the excess pore pressure

$$
\frac{d P_{f}^{\prime}}{d t}+\nabla \cdot\left(\frac{k}{\mu_{f} \alpha} \nabla P_{f}^{\prime}\right)=\frac{\dot{\gamma} \tan \psi}{\alpha}-\frac{d}{d t}\left(\Sigma_{y y}-\rho(h-y) \cos \theta-\mu_{f} \frac{\partial V}{\partial y}\right) .
$$

Although this equation looks similar to other formulations used for fluidized beds ${ }^{59}$ and soil mechanics, ${ }^{23}$ it differs in many respects. The most obvious difference lies in the forcing term, which shows how shear and variations in the stress field can generate pore pressure variations. More subtle is the definition of the pressure diffusivity, which is $D=k /\left(\mu_{f} \alpha\right)$ in Iverson's model where it is defined as $D=k /\left(\mu_{f} \delta\right)$ with $\delta$ the adiabatic fluid compressibility. As for ordinary fluids, $\delta \sim 10^{-10} \mathrm{~Pa}^{-1}$, compared with Iverson's estimate $\alpha \sim 10^{-5} \mathrm{~Pa}^{-1}$, thus there is a factor $10^{5}$ difference in the estimation of the diffusivity. Equation (A17) also differs from the equation used in other granular-flow models in that it includes a time derivative whereas in models such as that developed by Pailha and Pouliquen, ${ }^{60}$ the pressure adapts instantaneously to the seepage velocity.

Dilatancy and pore pressure play synergetic roles in the shear resistance and flow stability: for instance, if the bulk dilates, the expansion of pore space causes a pressure reduction. The normal traction on the granular skeleton $\Sigma_{p}^{y y}=\Sigma_{y y}-P_{f}$ is then increased, which in turn causes the shearstress to increase as $\Sigma_{p}^{x y}=K \Sigma_{p}^{y y}$. The balance between the driving and resisting forces leads to compaction and an increase in pore pressure. If the dilatancy and particle friction angles $\psi$ and $\varphi$ were independent, the steady-state fluid pressure would be

$$
P_{f}=\Sigma_{y y} \frac{\tan (\varphi+\psi)-\tan \theta}{\tan (\varphi+\psi)} .
$$

As $\psi$ is a function of the shear rate, this equation is implicit. Depending on the specific expression used to relate $\psi$ and $\dot{\gamma}$, it may admit a unique steady-state solution, along with unstable solutions, which may give rise to cyclic motion (plastic regime). ${ }^{20}$

If we consider a simplified dimensionless version of the pore pressure equation (A17) (nearly steady uniform layer of material), we obtain a linear diffusion equation with a forcing term, which may become vanishingly small:

$$
\frac{\partial P_{f}^{\prime}}{\partial t}+D \frac{\partial^{2} P_{f}^{\prime}}{\partial y^{2}}=\frac{\dot{\gamma} \tan \psi}{\alpha}
$$

where $D=k /\left(\mu_{f} \alpha\right)=3 \times 10^{-6} \mathrm{~m}^{2} / \mathrm{s}$, is the pore pressure diffusivity $\left(k=4 \times 10^{-12} \mathrm{~m}^{2}\right.$ is the permeability and $\alpha$ is the mixture compressibility $\alpha \sim 10^{-5} \mathrm{~Pa}^{-1}$ ). Equation (A19) shows that when shear and dilatancy act together $(\dot{\gamma}>0$ and $\tan \psi>0)$, excess pore pressure is governed by a Darcy-like process and pressure variations propagate instantaneously throughout the bulk. When the material reaches equilibrium ( $\tan \psi=0$ ), excess pore pressure diffuses throughout the bulk until it vanishes and the characteristic time of diffusion is $T_{D}=H_{*}^{2} / D=225 \mathrm{~s}$. Figure 2 shows that there were different phases in the time variation of the pore pressure: at short times $(t \ll 4 \mathrm{~min})$, the pore pressure adapted instantaneously to depth variations. In the stick-slip regime, we distinguished a short timescale $T_{s s} \sim 10 \mathrm{~s}$ corresponding to the pore pressure cycles and a longer timescale $T_{\text {relax }}$. $\sim 5 \mathrm{~min}$, which corresponded to the pore pressure relaxation, which did not seem to be controlled by depth variations. The value of this second timescale $T_{\text {relax }}$ matched the diffusion timescale $T_{D}$ approximately, while the shortest timescale $T_{s s}$ could be more consistent with a Darcy-like process. We thus conclude that the general picture and the estimates of the characteristic times provide some support for Iverson's approach. In the absence of particle concentration measurements, it 
was impossible to estimate the dilatancy and go further in the quantitative analysis of Eq. (A19). We emphasize that this qualitative agreement is a condition of the definition of the pore pressure diffusivity. If we had taken $\alpha=\delta=10^{-10} \mathrm{~Pa}^{-} 1$ as the adiabatic fluid compressibility, we would have found radically different estimates. Therefore this raises the important question concerning the origins of pore pressure fluctuations in suspensions.

\section{Boundary conditions}

In free-surface flows, deformation of the free boundary gives rise to capillary forces. Capillary normal forces resist the dilatancy-induced protrusion of particles from the boundary. The surface deformation of such a flowing suspension was documented by Loimer, Nir, and Semiat ${ }^{27}$ and Timberlake and Morris. ${ }^{29}$ The former authors estimated that the protrusion-resisting capillary force induces a normal stress at the free surface, which was approximated by

$$
\Sigma_{c a p}^{y y}=2 n \gamma \pi\left\langle 2 \ell-\frac{\ell^{2}}{a}\right\rangle,
$$

where $\ell$ is the protrusion height above the mean free-surface and $n$ is the density number. The protrusions also induce a jump in the fluid pressure

$$
\Delta p=\frac{\gamma}{r}
$$

with $r=O(a)$ the curvature radius of the meniscus in the pore space. Fall et al. ${ }^{9}$ and Cates, Haw, and Holmes $^{28}$ considered that for particle concentrations close to the maximum packing fraction, surface tension is sufficient to increase shear resistance and gives rise to an apparent yield stress. Using magnetic resonance imaging, Fall et al. ${ }^{9}$ investigated how the rheological properties depended on the density difference between the fluid and the particles. They found that a slight density contrast was necessary for a yield stress to arise. For perfectly density-matched suspensions, they observed no plastic behavior (except for very high concentrations, for which dilatancy imposed steric constraints).

${ }^{1}$ W. B. Russel, D. A. Saville, and W. R. Schowalter, Colloidal Dispersions (Cambridge University Press, Cambridge, 1995).

${ }^{2}$ C. R. Wildemuth and M. C. Williams, "A new interpretation of viscosity and yield stress in dense slurries: coal and other irregular particles,” Rheol. Acta 24, 75-91 (1985).

${ }^{3}$ D. M. Husband, N. Aksel, and W. Geissle, "The existence of static yield stresses in suspensions containing noncolloidal particles,” J. Rheol. 37, 215-235 (1993).

${ }^{4}$ L. Heymann, S. Peukert, and N. Aksel, "On the solid-liquid transition of concentrated suspensions in transient shear flow," Rheol. Acta 41, 307-315 (2002).

${ }^{5}$ J. Z. Q. Zhou, T. Fang, G. Luo, and P. H. T. Uhlherr, "Yield stress and maximum packing fraction of concentrated suspensions," Rheol. Acta 34, 544-561 (1995).

${ }^{6}$ C. Ancey, "Role of lubricated contacts in concentrated polydisperse suspensions," J. Rheol. 45, 1421-1439 (2001).

${ }^{7}$ M. Lenoble, P. Snabre, and B. Pouligny, "The flow of a very concentrated slurry in a parallel-plate device: Influence of gravity," Phys. Fluids 17, 073303 (2005).

${ }^{8}$ G. Ovarlez, F. Bertrand, and S. Rodts, "Local determination of the constitutive law of a dense suspension of noncolloidal particles through magnetic resonance imaging," J. Rheol. 50, 259-292 (2006).

${ }^{9}$ A. Fall, F. Bertrand, G. Ovarlez, and D. Bonn, "Yield stress and shear banding in granular suspensions," Phys. Rev. Lett. 103, 178301 (2009).

${ }^{10}$ S. Wiederseiner, N. Andreini, G. Épely-Chauvin, and C. Ancey, "Refractive index matching in concentrated particle suspensions: A review," Exp. Fluids 50, 1183-1206 (2011).

${ }^{11}$ S. Deboeuf, G. Gauthier, J. C. Martin, Y. Yurkovetsky, and J. F. Morris, "Particle pressure in a sheared suspension: A bridge from osmosis to granular dilatancy," Phys. Rev. Lett. 102, 108301 (2009).

${ }^{12}$ N. Andreini, "Dam break of Newtoniam fluids and granular suspensions: Internal dynamics measurements," Ph.D. dissertation, Ecole Polytechnique Fédérale de Lausanne, 2012.

${ }^{13}$ C. Ancey, S. Cochard, and N. Andreini, "The dam-break problem for viscous fluids in the high-capillary-number limit," J. Fluid Mech. 624, 1-22 (2009).

${ }^{14}$ C. Bonnoit, T. Darnige, E. Clément, and A. Lindner, "Inclined plane rheometry of a dense granular suspension," J. Rheol. 54, 65-79 (2010).

${ }^{15}$ N. Andreini, G. Epely-Chauvin, and C. Ancey, "Internal dynamics of Newtonian and viscoplastic fluid avalanches down a sloping bed," Phys. Fluids 24, 053101 (2012).

${ }^{16}$ B. Nsom, "The dam break problem for a hyperconcentrated suspension," Appl. Rheol. 10, 224-230 (2000).

${ }^{17}$ P. Bohorquez, "Finite volume method for falling liquid films carrying monodisperse spheres in Newtonian regime," AIChE J. 58, 2601-2616 (2012). 
${ }^{18}$ R. M. Iverson, “Landslide triggering by rain infiltration,” Water Resour. Res. 36, 1897-1910, doi:10.1029/2000WR900090 (2000).

${ }^{19}$ R. M. Iverson, "Debris-flow mechanics," in Debris-Flow Hazards and Related Phenomena, edited by M. Jakob and O. Hungr (Springer, Berlin, 2005), pp. 105-134.

${ }^{20}$ D. G. Schaeffer and R. M. Iverson, "Steady and intermittent slipping in a model of landslide motion regulated by porepressure feedback," SIAM J. Appl. Math. 69, 769-786 (2008).

${ }^{21}$ M. Pailha, M. Nicolas, and O. Pouliquen, "Initiation of underwater granular avalanches: Influence of the initial volume fraction," Phys. Fluids 20, 111701 (2008)

${ }^{22}$ S. D. Kulkarni, B. Metzger, and J. F. Morris, "Particle-pressure-induced self-filtration in concentrated suspensions," Phys. Rev. E 82, 010402(R) (2010).

${ }^{23}$ L. Goren, E. Aharonov, D. Sparks, and R. Toussaint, "The mechanical coupling of fluid-filled granular material under shear," Pure Appl. Geophys. 168, 2289-2323 (2011).

${ }^{24}$ R. M. Iverson, "Elements of an improved model of debris-flow motion," AIP Conf. Proc. 1145, 9-16 (2009).

${ }^{25}$ D. L. George, and R. M. Iverson, "A two-phase debris-flow model that includes coupled evolution of volume fractions, granular dilatancy, and pore-fluid pressure," in Proceedings of Fifth International Conference on Debris-flow Hazards Mitigation, Mechanics, Prediction and Assessment, edited by R. Genevois, D. L. Hamilton, and A. Prestinizi (Casa Editrice Universita La Sapienza, Rome, Padova, 2011), pp. 415-424.

${ }^{26}$ C. M. Carlevaro and L. A. Pugnaloni, "Arches and contact forces in a granular pile," Eur. Phys. J. E 35, 44 (2012).

${ }^{27}$ T. Loimer, A. Nir, and R. Semiat, "Shear-induced corrugation of free interfaces in concentrated suspensions," J. Non-Newtonian Fluid Mech. 102, 115-134 (2002).

${ }^{28}$ M. E. Cates, D. D. Haw, and C. B. Holmes, "Dilatancy, jamming, and the physics of granulation," J. Phys.: Condens. Matter 17, S2517-S2531 (2005).

${ }^{29}$ B. D. Timberlake and J. F. Morris, "Particle migration and free-surface topography in inclined plane flow of a suspension," J. Fluid Mech. 538, 309-341 (2005).

${ }^{30} \mathrm{E}$. Brown and H. M. Jaeger, "The role of dilation and confining stresses in shear thickening of dense suspensions," J. Rheol 56, 875-923 (2012).

${ }^{31}$ M. C. Jenkins, M. D. Haw, G. C. Barker, W. C. K. Poon, and S. U. Egelhaaf, "Does gravity cause load-bearing bridges in colloidal and granular systems?" Phys. Rev. Lett. 107, 038302 (2011).

${ }^{32}$ C. Bonnoit, J. Lanuza, A. Lindner, and E. Clément, "Mesoscopic length scale controls the rheology of dense suspensions," Phys. Rev. Lett. 105, 108302 (2010).

${ }^{33}$ M. D. Haw, "Jamming, two-fluid behavior, and "Self-filtration" in concentred particulate suspensions," Phys. Rev. Lett. 92, 185506 (2004)

${ }^{34}$ M. E. Cates, J. P. Wittmer, J. P. Bouchaud, and P. Claudin, "Jamming, force chains, and fragile matter," Phys. Rev. Lett. 81, 1841-1844 (1998).

${ }^{35}$ G. K. Batchelor, "The stress system in a suspension of free-force particles," J. Fluid Mech. 41, 545-570 (1970).

${ }^{36}$ Y. A. Buyevich and I. N. Shchelchkova, "Flow of dense suspension," Prog. Aerosp. Sci. 18, 121-150 (1979).

${ }^{37}$ R. Herczynski and I. Pienkowska, "Toward a statistical theory of suspension," Annu. Rev. Fluid Mech. 12, 237-269 (1980).

${ }^{38}$ D. Lhuillier, "Ensemble averaging in slightly non-uniform suspensions," Eur. J. Mech. B/Fluids 6, 649-661 (1992).

${ }^{39}$ D. A. Drew and S. L. Passman, Theory of Multicomponent Fluids (Springer, New York, 1999).

${ }^{40}$ J. F. Morris, "A review of microstructure in concentrated suspensions and its implications for rheology and bulk flow," Rheol. Acta 48, 909-923 (2009).

${ }^{41}$ P. R. Nott, E. Guazzelli, and O. Pouliquen, "The suspension balance model revisited,” Phys. Fluids 23, 043304 (2011).

${ }^{42}$ D. Lhuillier, "Migration of rigid particles in non-Brownian viscous suspensions," Phys. Fluids 21, 023302 (2009).

${ }^{43}$ P. R. Nott and J. F. Brady, "Pressure-driven flow of suspensions: simulation and theory," J. Fluid Mech. 275, 157-199 (1994).

${ }^{44}$ J. F. Morris and F. Boulay, "Curvilinear flows of noncolloidal suspensions: The role of normal stresses," J. Rheol. 43, 1213-1238 (1999).

${ }^{45}$ R. M. Miller, J. P. Singh, and J. F. Morris, "Suspension flow modeling for general geometries," Chem. Eng. Sci. 64, 4597-4610 (2009).

${ }^{46}$ I. E. Zarraga, D. A. Hill, and D. T. Leighton, "The characterization of the total stress of concentrated suspensions of noncolloidal spheres in Newtonian fluids," J. Rheol. 44, 185-221 (2000).

${ }^{47}$ N. A. Frankel and A. Acrivos, "On the viscosity of a concentrated suspension of solid spheres," Chem. Eng. Sci. 22, 847-853 (1967).

${ }^{48}$ J. D. Goddard, "An elastohydrodynamic theory for the rheology of concentrated suspensions of deformable particles," J. Non-Newtonian Fluid Mech. 2, 169-189 (1977).

${ }^{49}$ B. H. A. A. van der Brule and R. J. J. Jongshaap, "Modeling of concentrated suspensions," J. Stat. Phys. 62, 1225-1237 (1991).

${ }^{50}$ C. Ancey, P. Coussot, and P. Evesque, "A theoretical framework for very concentrated granular suspensions in a steady simple shear flow," J. Rheol. 43, 1673-1699 (1999).

${ }^{51}$ N. Phan-Thien, X. J. Fan, and B. C. Khoo, "A new constitutive model for monodispersed suspensions of spheres at high concentrations," Rheol. Acta 38, 297-304 (1999).

52 J. J. Stickel, R. J. Phillips, and R. L. Powell, "A constitutive model for microstructure and total stress in particulate suspensions," J. Rheol. 50, 379-413 (2006).

${ }^{53}$ E. Bertrand, J. Bibette, and V. Schmitt, "From shear thickening to shear-induced jamming," Phys. Rev. E 66, 060401(R) (2002).

${ }^{54}$ D. Leighton and A. Acrivos, "Viscous resuspension," Chem. Eng. Sci. 41, 1377-1384 (1986).

${ }^{55}$ C. Ancey and P. Coussot, "Transition from frictional to viscous regime for granular suspensions," C. R. Acad. Sci. Paris sér. IIb 327, 515-522 (1999). 
${ }^{56}$ P. Coussot and C. Ancey, "Rheophysical classification of concentrated suspensions and granular pastes," Phys. Rev. E 59, 4445-4457 (1999).

${ }^{57}$ F. Boyer, E. Guazzelli, and O. Pouliquen, "Unifying suspension and granular rheology," Phys. Rev. Lett. 107, 188301 (2011).

${ }^{58}$ M. A. Van der Hoef, R. Beetstra, and J. A. M. Kuipers, "Lattice-Boltzmann simulations of low-Reynolds-number flow past mono- and bidisperse arrays of spheres: Results for the permeability and drag force," J. Fluid Mech. 528, 233-254 (2005).

${ }^{59}$ S. Montserrat, A. Tamburrino, O. Roche, and Y. Niño, "Pore fluid pressure diffusion in defluidizing granular columns," J. Geophys. Res. 117, F02034, doi:10.1029/2011JF002164 (2012).

${ }^{60}$ M. Pailha and O. Pouliquen, "A two-phase flow description of the initiation of underwater granular avalanches," J. Fluid Mech. 633, 115-135 (2009).

${ }^{61}$ C. Ancey, N. Andreini, and G. Epely-Chauvin, "Granular suspension avalanches. I. Macro-viscous behavior," Phys. Fluids 25, 033301 (2013). 